\title{
DEVIATION INEQUALITIES FOR BANACH SPACE VALUED MARTINGALES DIFFERENCES SEQUENCES AND RANDOM FIELDS
}

\author{
DAVIDE GIRAUDO
}

\begin{abstract}
We establish deviation inequalities for the maxima of partial sums of a martingale differences sequence, and of an orthomartingale differences random field. These inequalities can be used to give rates for linear regression and the law of large numbers.
\end{abstract}

\section{INTRODUCTION AND MAIN RESULTS}

Deviation inequalities play an important role in the study of properties of partial sums of random variables. A particular attention has been given to martingales. In Burkholder's paper [Bur73], distribution function inequalities for maximum of martingales are established, and moment inequalities are derived from them. Sharp results has been obtained for martingales with bounded increments [Hoe63, Azu67]. When the increments of the considered martingale are unbounded but square integrable, it is possible to control the tail function of the martingale by that of the increments and of the sum of conditional variances, like in [Bur73, Hae84, dlPn99, FGL12, FGL15]. When the tail of increments have a polynomial decay, it seems that Nagaev's inequality [Nag03] gives the most satisfactory results. It states the following: for any positive $q$, there exists a constant $C(q)$ such that if $\left(S_{n}\right)_{n \geqslant 1}$ is a martingale defined on a probability space $(\Omega, \mathcal{F}, \mathbb{P})$ and $X_{i}:=S_{i}-S_{i-1}$, then

$$
\begin{aligned}
\mathbb{P}\left\{\left|S_{n}\right|>x\right\} \leqslant C(q) \int_{0}^{1} \mathbb{P}\left\{\max _{1 \leqslant i \leqslant n}\left|X_{i}\right|>x u\right\} u^{q-1} \mathrm{~d} u & \\
& +C(q) \int_{0}^{1} \mathbb{P}\left\{\left(\sum_{i=1}^{n} \mathbb{E}\left[X_{i}^{2} \mid \mathcal{F}_{i-1}\right]\right)>x u\right\} u^{q-1} \mathrm{~d} u .
\end{aligned}
$$

The constant $C(q)$ is of order $e^{e^{q}}$. The result (without the absolute valued in the left hand side of (1.1)) holds for supermartingales. There are three possibilities of improvement of the version of Nagaev's result for martingales:

- the result can only be used for square integrable martingales. One can wonder whether a similar inequality as (1.1) holds when $X_{i} \in \mathbb{L}^{p}$ where $1<p<2$.

- In [Nag03], the real valued case is considered, and the proof suggests that the extension to the Banach valued case is challenging.

- Finally, the improvement of the constant $C(q)$ is also of interest.

Let us explain the idea of proof of an extension of (1.1) (see Theorem 1.3) in the real valued case, with square integrability. Define

$$
\begin{gathered}
f(x):=\mathbb{P}\left\{\left|S_{n}\right|>x\right\} \text { and } \\
g(x):=\mathbb{P}\left\{\max _{1 \leqslant i \leqslant n}\left|X_{i}\right|>x\right\}+\mathbb{P}\left\{\left(\sum_{i=1}^{n} \mathbb{E}\left[X_{i}^{2} \mid \mathcal{F}_{i-1}\right]\right)>x\right\},
\end{gathered}
$$

Date: August 30, 2018.

Key words and phrases. Martingales, random fields, orthomartingales, deviation inequalities, complete convergence. 
We prove in Lemma 3.1 that for any positive $x$.

$$
f(2 x) \leqslant \delta^{2}(1-\delta)^{-2} f(x)+g(\delta x) .
$$

This is done by using a martingale transform of the original martingale, the former having small conditional variances. Using monotonicity of the function $g,(1.4)$ can be converted into an integral inequality.

The paper is organized as follows: in Subsection 1.1, we state a deviation inequality for any Banach space valued martingale differences sequence, then for stochastically dominated or identically distributed sequences. In Subsection 1.2, we review orthomartingales, and state a deviation inequality for orthomartingale differences random fields. Section 2 is devoted to applications to linear regression and Baum-Katz estimates martingale differences sequence and orthomartingale differences random fields. All these results are proven in Section 3.

\subsection{Martingale differences sequences.}

\subsubsection{General case.}

Definition 1.1. Let $(\Omega, \mathcal{F}, \mathbb{P})$ be a probability space and let $\left(B,\|\cdot\|_{B}\right)$ be a separable Banach space. For any $p \geqslant 1$, we denote by $\mathbb{L}_{B}^{p}$ the space of B-valued random variables such that $\|X\|_{\mathbb{L}_{B}^{p}}^{p}=\mathbb{E}\left[\|X\|^{p}\right]$ is finite. Let $\left(\mathcal{F}_{i}\right)_{i \geqslant 1}$ be an non-decreasing sequence of sub- $\sigma$-algebras of $\mathcal{F}$. We say that a sequence of $B$-valued random variables $\left(X_{i}\right)_{i \geqslant 1}$ is a martingale differences sequence with respect to the filtration $\left(\mathcal{F}_{i}\right)_{i \geqslant 1}$ if

(1) for any $i \geqslant 1, X_{i}$ is $\mathcal{F}_{i}$-measurable and belongs to $\mathbb{L}_{B}^{1}$;

(2) for any $i \geqslant 2, \mathbb{E}\left[X_{i} \mid \mathcal{F}_{i-1}\right]=X_{i-1}$ almost surely.

Definition 1.2. Following [Pis75], we say that a Banach space $(B,\|\cdot\|)$ is $r$-smooth $(1<r \leqslant 2)$ if there exists an equivalent norm $\|\cdot\|^{\prime}$ such that

$$
\sup _{t>0} \frac{1}{t^{r}} \sup \left\{\|x+t y\|^{\prime}+\|x-t y\|^{\prime}-2:\|x\|^{\prime}=\|y\|^{\prime}=1\right\}<\infty .
$$

From [Ass75], we know that if $B$ is $r$-smooth and separable, then there exists a constant $D$ such that for any sequence of $B$-valued martingale differences $\left(X_{i}\right)_{i \geqslant 1}$,

$$
\mathbb{E}\left[\left\|\sum_{i=1}^{n} X_{i}\right\|^{r}\right] \leqslant D \sum_{i=1}^{n} \mathbb{E}\left[\left\|X_{i}\right\|^{r}\right] .
$$

Since an $r$-smooth Banach space is also $r^{\prime}$-smooth for any $1<r^{\prime} \leqslant r$, there exists a constant $C_{r^{\prime}, B}$ such that such that for any sequence of $B$-valued martingale differences $\left(X_{i}\right)_{i \geqslant 1}$, and any integer $n$,

$$
\mathbb{E}\left[\left\|\sum_{i=1}^{n} X_{i}\right\|^{r^{\prime}}\right] \leqslant C_{r^{\prime}, B} \sum_{i=1}^{n} \mathbb{E}\left[\left\|X_{i}\right\|^{r^{\prime}}\right] .
$$

Our first main result is an inequality in the spirit of Theorem 1 in [Nag03].

Theorem 1.3. Let $(B,\|\cdot\|)$ be a separable $r$-smooth Banach space where $1<r \leqslant 2$. For each $1<r^{\prime} \leqslant r, q>0$ and for any B-valued martingale differences sequence $\left(X_{i}, \mathcal{F}_{i}\right)_{i \geqslant 1}$, the following 
inequality holds for each $n \geqslant 1$ and $x>0$ :

$$
\begin{aligned}
\mathbb{P}\left\{\max _{1 \leqslant i \leqslant n}\left\|S_{i}\right\|>x\right\} \leqslant \frac{2^{q}}{2^{q}-1} q 2^{-r^{\prime}} \int_{0}^{1} \mathbb{P}\left\{\max _{1 \leqslant i \leqslant n}\left\|X_{i}\right\|>2^{-1-q / r^{\prime}} C_{r^{\prime}, B}^{-1 / r^{\prime}} x u\right\} u^{q-1} \mathrm{~d} u \\
+\frac{2^{q}}{2^{q}-1} q 2^{-r^{\prime}} \int_{0}^{1} \mathbb{P}\left\{\left(\sum_{i=1}^{n} \mathbb{E}\left[\left\|X_{i}\right\|^{r^{\prime}} \mid \mathcal{F}_{i-1}\right]\right)^{1 / r^{\prime}}>2^{-1-q / r^{\prime}} C_{r^{\prime}, B}^{-1 / r^{\prime}} x u\right\} u^{q-1} \mathrm{~d} u
\end{aligned}
$$

where $S_{i}=\sum_{i=1}^{n} X_{i}$ and $C_{r^{\prime}, B}$ is a constant satisfying (1.6) for any $n$ and any martingale differences sequence.

Remark 1.4. On one hand, Nagaev's result [Nag03] applies to real valued supermartingales, while our result is restricted to martingales. On the other hand, when applied to the latter class of random variable, our result gives a generalization in two directions. First, we consider Banach space valued random variables. Second, even when restricted to real-valued random variables, our result can be used to treat martingales whose increments do not necessarily have a finite moment of order 2 .

In the independent setting, the terms $\mathbb{E}\left[\left\|X_{i}\right\|^{r^{\prime}} \mid \mathcal{F}_{i-1}\right]$ are constant hence we can state the following Corollary of Theorem 1.3.

Corollary 1.5. Let $(B,\|\cdot\|)$ be a separable $r$-smooth Banach space where $1<r \leqslant 2$. For each $1<r^{\prime} \leqslant r, q>0$ and for any independent centered sequence $\left(X_{i}\right)_{i \geqslant 1}$, the following inequality holds for each $n \geqslant 1$ and $x>0$ :

$$
\begin{array}{r}
\mathbb{P}\left\{\max _{1 \leqslant i \leqslant n}\left\|S_{i}\right\|>x\right\} \leqslant \frac{2^{q}}{2^{q}-1} q 2^{-r^{\prime}} \int_{0}^{1} \mathbb{P}\left\{\max _{1 \leqslant i \leqslant n}\left\|X_{i}\right\|>2^{-1-q / r^{\prime}} C_{r^{\prime}, B}^{-1 / r^{\prime}} x u\right\} u^{q-1} \mathrm{~d} u \\
+C_{r^{\prime}, B}^{(q-1) / r^{\prime}} \frac{2^{q}}{2^{q}-1} 2^{-r^{\prime}} 2^{q+q^{2} / r^{\prime}} x^{-q}\left(\sum_{i=1}^{n} \mathbb{E}\left[\left\|X_{i}\right\|^{r^{\prime}}\right]\right)^{q / r^{\prime}},
\end{array}
$$

where $S_{i}=\sum_{i=1}^{n} X_{i}$ and $C_{r^{\prime}, B}$ is a constant satisfying (1.6) for any $n$ and any martingale differences sequence.

1.1.2. Stochastically dominated sequences. For a random variable $Y$ with values in the Banach space $(B,\|\cdot\|)$, we denote by $Q_{Y}$ the generalized inverse of the function $t \mapsto \mathbb{P}\{\|Y\|>t\}$, that is,

$$
Q_{Y}(u):=\inf \{t>0 \mid \mathbb{P}\{\|Y\|>t\} \leqslant u\}, \quad u \in[0,1] .
$$

Definition 1.6. Let $\left(X_{i}\right)_{i \geqslant 1}$ be a sequence of random variables with values in a Banach space $(B,\|\cdot\|)$ and let $X: \Omega \rightarrow \mathbb{R}$ be a real valued random variable. We say that $\left(X_{i}\right)_{i \geqslant 1} \prec X$ if for all $u \in[0,1]$, and any $i \geqslant 1, Q_{X_{i}}(u) \leqslant Q_{X}(u)$.

In the case where the random variables $X_{i}, 1 \leqslant i \leqslant n$ are stochastically dominated and $\mathbb{E}\left[\left\|X_{i}\right\|^{r^{\prime}} \mid \mathcal{F}_{i-1}\right]$ bounded by identically distributed random variables, the result of Theorem 1.3 admits the following simplification.

Theorem 1.7. Let $(B,\|\cdot\|)$ be an $r$-smooth separable Banach space. For each $1<r^{\prime} \leqslant r$ and each $q>r^{\prime}$, for any martingale differences sequence $\left(X_{i}, \mathcal{F}_{i}\right)_{i \geqslant 0}$ with values in $B$ such that there exists real valued random variables $X$ and $V_{i}, i \geqslant 1$ for which $\left(X_{i}\right)_{i \geqslant 1} \prec X, \mathbb{E}\left[\left\|X_{i}\right\|^{r^{\prime}} \mid \mathcal{F}_{i-1}\right] \leqslant V_{i}$ a.s. and 
$\left(V_{i}\right)_{i \geqslant 1}$ is identically distributed, then the following inequality hold for any $n \geqslant 1$ and $x>0$ :

$$
\begin{aligned}
& \mathbb{P}\left\{\max _{1 \leqslant i \leqslant n}\left\|S_{i}\right\|>\right.\left.x n^{1 / r^{\prime}}\right\} \leqslant \frac{2^{q}}{2^{q}-1} q 2^{-r^{\prime}} n \int_{0}^{1} \mathbb{P}\left\{X>2^{-1-q / r^{\prime}} C_{r^{\prime}, B}^{-1 / r^{\prime}} x u n^{1 / r^{\prime}}\right\} u^{q-1} \mathrm{~d} u \\
&+\frac{2^{q}}{2^{q}-1} \frac{q 2^{-r^{\prime}}}{q-r^{\prime}} \int_{0}^{+\infty} \mathbb{P}\left\{V_{1}>2^{-2-q / r^{\prime}} C_{r^{\prime}, B}^{-1 / r^{\prime}} x^{r^{\prime}} w\right\} \min \left\{w^{\frac{q-r^{\prime}}{r^{\prime}}}, 1\right\} \mathrm{d} w .
\end{aligned}
$$

If the sequence $\left(\left\|X_{i}\right\|\right)_{i \geqslant 1}$ is identically distributed, then for any $n \geqslant 1$ and $x>0$ :

$$
\mathbb{P}\left\{\max _{1 \leqslant i \leqslant n}\left\|S_{i}\right\|>x n^{1 / r^{\prime}}\right\} \leqslant \frac{2^{q+1}}{2^{q}-1} \frac{q 2^{-r^{\prime}}}{q-r^{\prime}} \int_{0}^{+\infty} \mathbb{P}\left\{\left\|X_{1}\right\|>x u\right\} \min \left\{u^{q-1}, u^{r^{\prime}-1}\right\} \mathrm{d} u,
$$

where $S_{i}=\sum_{j=1}^{i} X_{j}$.

1.2. Orthomartingale differences random fields. The results of the previous section can be extended in some sense to random fields, that is, processes indexed by $\mathbb{N}^{d}$ or $\mathbb{Z}^{d}$ where $d \geqslant 1$ be an integer. In order to state them, we have to give a precise definition of martingales in this setting.

We use the following notations:

(1) for $\mathbf{i}=\left(i_{q}\right)_{q=1}^{d}$ and $\mathbf{j}=\left(j_{q}\right)_{q=1}^{d}$ we write $\mathbf{i} \preccurlyeq \mathbf{j}$ if and only if $i_{q} \leqslant j_{q}$ for all $q \in\{1, \ldots, d\}$;

(2) if $\mathbf{k}$ and $\mathbf{l} \in \mathbb{Z}^{d}$ the coordinatewise minimum is defined by $\min \{\mathbf{k}, \mathbf{l}\}=\left(\min \left\{k_{i}, l_{i}\right\}\right)_{i=1}^{d}$.

(3) The addition is defined coordinatewise.

(4) If $\mathbf{n}=\left(n_{q}\right)_{q=1}^{d}$ is an element of $\mathbb{N}^{d}$, then $|\mathbf{n}|$ denotes $\prod_{q=1}^{d} n_{q}$.

(5) For $j \in\{1, \ldots, d\}, \mathbf{e}_{\mathbf{j}}$ denotes the element of $\mathbb{Z}^{d}$ whose $j$-th coordinate is 1 and all the others are zero. Moreover, $\mathbf{1}$ is the element of $\mathbb{Z}^{d}$ whose all coordinates are 1.

Definition 1.8. The family $\left(\mathcal{F}_{\mathbf{i}}\right)_{\mathbf{i} \in \mathbb{Z}^{d}}$ of sub- $\sigma$-algebras of $\mathcal{F}$ is a filtration if $\mathcal{F}_{\mathbf{i}} \subset \mathcal{F}_{\mathbf{j}}$ whenever $\mathbf{i} \preccurlyeq \mathbf{j}$.

Definition 1.9. Let $\left(\mathcal{F}_{\mathbf{i}}\right)_{\mathbf{i} \in \mathbb{Z}^{d}}$ be a filtration. If for each $\mathbf{i}, \mathbf{j} \in \mathbb{Z}^{d}$ and each integrable random variable $Y$,

$$
\mathbb{E}\left[\mathbb{E}\left[Y \mid \mathcal{F}_{\mathbf{i}}\right] \mid \mathcal{F}_{\mathbf{j}}\right]=\mathbb{E}\left[\mathbb{E}\left[Y \mid \mathcal{F}_{\mathbf{j}}\right] \mid \mathcal{F}_{\mathbf{i}}\right]=\mathbb{E}\left[Y \mid \mathcal{F}_{\min \{\mathbf{i}, \mathbf{j}\}}\right] \text { almost surely, }
$$

the filtration $\left(\mathcal{F}_{\mathbf{i}}\right)_{\mathbf{i} \in \mathbb{Z}^{d}}$ is said to be commuting.

Definition 1.10. The collection of random variables $\left\{M_{\mathbf{n}}, \mathbf{n} \in \mathbb{Z}^{d}\right\}$ is said to be an orthomartingale random field with respect to the commuting filtration $\left(\mathcal{F}_{\mathbf{i}}\right)_{\mathbf{i} \in \mathbb{Z}^{d}}$ if for each $\mathbf{n} \in \mathbb{N}^{d}, M_{\mathbf{n}}$ is $\mathcal{F}_{\mathbf{n}}$-measurable, integrable and for each $\mathbf{i}, \mathbf{j} \in \mathbb{Z}^{d}$ such that $\mathbf{i} \preccurlyeq \mathbf{j}$,

$$
\mathbb{E}\left[M_{\mathbf{j}} \mid \mathcal{F}_{\mathbf{i}}\right]=M_{\mathbf{i}}
$$

Definition 1.11. The collection of random variables $\left(X_{\mathbf{i}}\right)_{\mathbf{i} \in \mathbb{Z}^{d}}$ is said to be an orthomartingale diffrences random field with respect to the commuting filtration $\left(\mathcal{F}_{\mathbf{i}}\right)_{\mathbf{i} \in \mathbb{Z}^{d}}$ if the random field $\left(S_{\mathbf{n}}\right)_{\mathbf{n} \in \mathbb{Z}^{d}}$ defined by

$$
S_{\mathbf{n}}:= \begin{cases}\sum_{\mathbf{1} \preccurlyeq \mathbf{i} \preccurlyeq \mathbf{n}} X_{\mathbf{i}} & \text { if } \mathbf{n} \succcurlyeq \mathbf{1}, \\ 0 & \text { otherwise, }\end{cases}
$$

is an orthomartingale random field with respect to the filtration $\left(\mathcal{F}_{\mathbf{i}}\right)_{\mathbf{i} \in \mathbb{Z}^{d}}$.

In all this subsection, we shall make the following assumption on the random field $\left(X_{\mathbf{i}}\right)_{\mathbf{i} \in \mathbb{Z}^{d}}$, namely:

$$
\text { for all } \mathbf{n} \succcurlyeq \mathbf{1}, \mathbf{l} \in \mathbb{Z}^{d},\left\|\sum_{\mathbf{1} \preccurlyeq \mathbf{i} \preccurlyeq \mathbf{n}} X_{\mathbf{i}}\right\| \text { and }\left\|\sum_{\mathbf{1} \preccurlyeq \mathbf{i} \preccurlyeq \mathbf{n}} X_{\mathbf{i}+\mathbf{1}}\right\| \text { have the same distribution. }
$$

Orthomartingale random fields have good properties with respect to marginal filtrations $\mathcal{F}_{q}^{(d)}:=$ $\sigma\left(\mathcal{F}_{\mathbf{k}}, k_{q} \leqslant j, \mathbf{k} \in \mathbb{Z}^{d}\right), q \in\{1, \ldots, d\}$. Furthermore, when a coordinate is fixed, we still have an orthomartingale random field with respect to a commuting filtration (see [Kho02], p.37, Theorem 3.5.1). 
Lemma 1.12. Let $\left(X_{\mathbf{i}}\right)_{\mathbf{i} \in \mathbb{Z}^{d}}$ be an orthomartingale difference random field with respect to the commuting filtration $\left(\mathcal{F}_{\mathbf{i}}\right)_{\mathbf{i} \in \mathbb{Z}^{d}}$, with values in a separable Banach space $(X,\|\cdot\|)$. Then the following properties hold.

(P.1) For any $\mathbf{n}=\left(n_{1}, \ldots, n_{d-1}\right) \in \mathbb{N}^{d-1}$, the sequence $\left(S_{(\mathbf{n}, j)}\right)_{j \geqslant 0}$ is a martingale with respect to the filtration $\left(\mathcal{F}_{j}^{(d)}\right)_{j \geqslant 0}$.

(P.2) For any $\mathbf{n}=\left(n_{1}, \ldots, n_{d-1}\right) \in \mathbb{N}^{d-1}$, the sequence $\left(\max _{\mathbf{i} \in[\mathbf{1}, \mathbf{n}]}\left\|S_{(\mathbf{i}, j)}\right\|\right)_{j \geqslant 0}$ is a non-negative submartingale with respect to the filtration $\left(\mathcal{F}_{j}^{(d)}\right)_{j \geqslant 0}$.

(P.3) For any $j \in \mathbb{N}$, the random field $\left(S_{(\mathbf{n}, j)}\right)_{\mathbf{n} \succcurlyeq \mathbf{1}}$ is an orthomartingale with respect to the commuting filtration $\left(\mathcal{F}_{\mathbf{i}}^{\prime}\right)_{\mathbf{i} \in \mathbb{Z}^{d-1}}$, where $\mathcal{F}_{\mathbf{0}}^{\prime}$ is the $\sigma$-algebra generated by $\bigcup_{l \in \mathbb{Z}} \mathcal{F}_{l \mathbf{e}_{\mathbf{d}}}$.

We now state the analogue of Theorem 1.7 for strictly stationary orthomartingale differences random fields.

Theorem 1.13. Let $(B,\|\cdot\|)$ be an $r$-smooth separable Banach space. For each $1<r^{\prime} \leqslant r, q>r^{\prime}$ and positive integer $d$, there exist a constant $C$ depending only on $r^{\prime}, q, d$ and $B$ such that for any strictly stationary orthomartingale differences random field $\left(X_{\mathbf{i}}, \mathcal{F}_{\mathbf{i}}\right)_{i \in \mathbb{Z}^{d}}$ with values in $B$, the following inequality holds for any $\mathbf{n} \in \mathbb{N}^{d}$ and $x>0$ :

$$
\mathbb{P}\left\{\max _{\mathbf{1} \preccurlyeq \mathbf{i} \preccurlyeq \mathbf{n}}\left\|S_{\mathbf{i}}\right\|>x|\mathbf{n}|^{1 / r^{\prime}}\right\} \leqslant C \int_{0}^{+\infty} \mathbb{P}\left\{\left\|X_{1}\right\|>x u\right\} \min \left\{u^{q-1}, u^{r^{\prime}-1}\right\}(1+|\log u|)^{d-1} \mathrm{~d} u .
$$

Remark 1.14. One can integrate the previously obtained inequalities to get moment inequalities. For example, it is possible to recover a multidimensional Burkholder-like inequality in the stationary case, like in [Faz05]. Like in the one dimensional case, it is also possible to establish inequalities in weak $\mathbb{L}^{p}$ spaces like in [JS88], Remark 6.

\section{Applications}

2.1. Linear regression. We consider the stochastic linear regression model given by

$$
X_{k}=\theta \phi_{k}+\varepsilon_{k}, \quad 1 \leqslant k \leqslant n,
$$

where

- $\left(X_{k}\right)_{1 \leqslant k \leqslant n}$ are the observations,

- $\left(\phi_{k}\right)_{1 \leqslant k \leqslant n}$ are the regression variables and

- $\left(\varepsilon_{k}\right)_{1 \leqslant k \leqslant n}$ the driven noises.

We shall make the following assumptions:

(A.1) the sequence $\left(\phi_{k}\right)_{1 \leqslant k \leqslant n}$ is independent;

(A.2) the $\sigma$-algebra generated by $\phi_{k}, 1 \leqslant k \leqslant n$ is independent of the $\sigma$-algebra generated by $\varepsilon_{k}$, $1 \leqslant k \leqslant n$

(A.3) For each $k \in\{2, \ldots, n\}, \mathbb{E}\left[\varepsilon_{k} \mid \sigma\left(\varepsilon_{i}, 1 \leqslant i \leqslant k-1\right)\right]=0$ and $\mathbb{E}\left[\varepsilon_{1}\right]=0$.

Let $\theta_{n}$ be the least square estimator defined by

$$
\theta_{n}:=\frac{\sum_{k=1}^{n} \phi_{k} X_{k}}{\sum_{i=1}^{n} \phi_{i}^{2}}
$$

Theorem 2.1. Suppose that the assumptions (A.1), (A.2) and (A.3) hold. Suppose that there exists constant $C_{1}$ and $C_{2}$ such that for any $i \in\{1, \ldots, n\}$,

$$
\mathbb{E}\left[\left|\varepsilon_{i}\right|^{p}\right] \leqslant C_{1} \text { and } \mathbb{E}\left[\varepsilon_{i}^{2} \mid \sigma\left(\varepsilon_{j}, 1 \leqslant j \leqslant i-1\right)\right] \leqslant C_{2} \text { a.s. }
$$


Then for any $p>2, q>p$ and any $x>0$,

$$
\mathbb{P}\left\{\left|\theta_{n}-\theta\right| \sqrt{\sum_{i=1}^{n} \phi_{i}^{2}}>x\right\} \leqslant C_{1} \frac{2^{q-2}}{2^{q}-1} \frac{q}{q-p} 2^{p+p q / 2} x^{-p}+\frac{2^{q-2}}{2^{q}-1} q 2^{q+q^{2} / 2} x^{-q} C_{2}^{q / 2} .
$$

Let us compare Theorem 2.1 with the results in [FGL17]. When $x$ is large, Theorem 2.1 and Theorems 3.3 and 3.4 in [FGL17] give an upper bound of order $x^{-p}$.

(1) In Theorem 3.3 of [FGL17], it is assumed that $\sup _{i}\left\|\mathbb{E}\left[\left|\varepsilon_{i}\right|^{p} \mid \sigma\left\{\varepsilon_{k}, 1 \leqslant k \leqslant i-1\right\}\right]\right\|_{\infty}<+\infty$, which is more restrictive than the assumption in Theorem 2.1.

(2) In Theorem 3.4 of [FGL17], $\sup _{i}\left\|\mathbb{E}\left[\left|\varepsilon_{i}\right|^{2} \mid \sigma\left\{\varepsilon_{k}, 1 \leqslant k \leqslant i-1\right\}\right]\right\|_{\infty}<+\infty$ and that there exists a positive $\delta$ and $C_{1}$ such that for all $i \geqslant 1, \mathbb{E}\left[\left|\varepsilon_{i}\right|^{p+\delta}\right] \leqslant C_{1}$, which is more restrictive than our result, since only boundedness of the sequence of moments of order $p$ is required.

\subsection{Baum-Katz estimates for martingale differences sequences and orthomartingale dif-} ferences random fields.

2.2.1. Martingale differences sequences. For $p>1$, we denote by $\mathbb{L}^{p, \infty}$ (respectively $\mathbb{L}_{0}^{p, \infty}$ ) the set of random variables $X$ such that $\sup _{t>0} t^{p} \mathbb{P}\{\|X\|>t\}<+\infty$ (respectively $\left.\lim _{t \rightarrow+\infty} t^{p} \mathbb{P}\{\|X\|>t\}=0\right)$. We also write $\mathbb{L}^{p} \log ^{q} \mathbb{L}$ (with $q \geqslant 0$ ) the set of random variables $X$ such that $\mathbb{E}\left[\|X\|^{p}\left(\log _{+}\|X\|\right)^{q}\right]$ is finite, where $\log _{+}(x):=\max \{0, \log \|x\|\}$.

Theorem 2.2. Let $B$ be an $r$-smooth Banach space for $1<r \leqslant 2$. Let $\left(X_{i}\right)_{i \geqslant 1}$ be a martingale differences sequence with values in B. Assume that one of the following conditions is satisfied:

(C.1) there exists a real valued random variable $X$ in $\mathbb{L}^{r}$ such that $\left(X_{i}\right)_{i \geqslant 1} \prec X$ and there exists an identically distributed sequence $\left(V_{i}\right)_{i \geqslant 1}$ such that for all $i, \mathbb{E}\left[\left\|X_{i}\right\|^{r} \mid \mathcal{F}_{i-1}\right] \leqslant V_{i}$ a.s. and $V_{i} \in \mathbb{L} \log \mathbb{L}$.

(C.2) The sequence $\left(\left\|X_{i}\right\|\right)_{i \geqslant 1}$ is identically distributed and $X_{1} \in \mathbb{L}^{r} \log \mathbb{L}$.

Then for each $\alpha \in(1 / r, 1]$ and each positive $x$, the series $\sum_{n=1}^{+\infty} n^{r \alpha-2} \mathbb{P}\left\{\max _{1 \leqslant i \leqslant n}\left\|S_{i}\right\|>n^{\alpha} x\right\}$ converges.

Let us compare this result with a previous one. In [DM07], convergence of the series

$$
\sum_{n=1}^{+\infty} n^{p \alpha-2} \mathbb{P}\left\{\max _{1 \leqslant i \leqslant n}\left\|S_{i}\right\|>n^{\alpha} x\right\}
$$

have been established for sequences satisfying $\left(X_{i}\right)_{i \geqslant 1} \prec X$ and $X \in \mathbb{L}^{p}$ for $1<p<r$ and $1 \leqslant \alpha \leqslant p$. Our result deal with a more restrictive class of martingale differences but covers the case $p=r$.

When a moment of order greater than two is finite, we can formulate precise results in terms of integrability of the increments and of the conditional variance term.

Theorem 2.3. Let $p>2,1 / 2<\alpha \leqslant 1$ and let $B$ be a separable 2 -smooth Banach space. There exists a constant $C(p, B)$ such that the following holds: for each $B$-valued martingale differences sequence $\left(X_{i}, \mathcal{F}_{i}\right)$ such that $\left(X_{i}\right) \prec X$ and such that there exists an identically distributed sequence $\left(V_{i}\right)_{i \geqslant 1}$ for which $\mathbb{E}\left[X_{i}^{2} \mid \mathcal{F}_{i-1}\right] \leqslant V_{i}$,

(1) if $X \in \mathbb{L}^{p / 2+1, \infty}$ and $V_{i} \in \mathbb{L}^{p / 2, \infty}$, then for each $x>0$,

$$
\begin{aligned}
\sup _{n \geqslant 1} n^{p(\alpha-1 / 2)} \mathbb{P}\left\{\max _{1 \leqslant i \leqslant n}\left\|S_{i}\right\|>n^{\alpha} x\right\} & \\
& \leqslant C(p, B)\left(\sup _{t>0} t^{p / 2+1} \mathbb{P}\left\{\left\|X_{1}\right\|>t\right\} x^{-p / 2-1}+\sup _{t>0} t^{p / 2} \mathbb{P}\left\{V_{1}>t\right\} x^{-p}\right) ;
\end{aligned}
$$


(2) if $X_{1} \in \mathbb{L}_{0}^{p / 2+1, \infty}$ and $V_{1} \in \mathbb{L}^{p / 2, \infty}$, then for each $x>0$,

$$
\lim _{n \rightarrow+\infty} n^{p(\alpha-1 / 2)} \mathbb{P}\left\{\max _{1 \leqslant i \leqslant n}\left\|S_{i}\right\|>n^{\alpha} x\right\}=0 ;
$$

(3) if $X_{1} \in \mathbb{L}^{p / 2+1}$ and $V_{1} \in \mathbb{L}^{p / 2}$, then

$$
\sum_{n=1}^{+\infty} n^{p(\alpha-1 / 2)-1} \mathbb{P}\left\{\max _{1 \leqslant i \leqslant n}\left\|S_{i}\right\|>n^{\alpha} x\right\} \leqslant C(p, B) x^{-p}\left(\left\|X_{1}\right\|_{p}^{p}+\left\|V_{1}\right\|_{p / 2}^{p / 2}\right) .
$$

We can formulate an analogous result for "norm-identically" distributed sequences.

Theorem 2.4. Let $p>2,1 / 2<\alpha \leqslant 1$ and let $B$ be a separable 2-smooth Banach space. There exists a constant $C(p, B)$ such that the following holds: for each B-valued martingale differences sequence $\left(X_{i}, \mathcal{F}_{i}\right)$ such that $\left(\left\|X_{i}\right\|\right)_{i \geqslant 1}$ is identically distributed,

(1) if $X_{1} \in \mathbb{L}^{p, \infty}$ then for each $x>0$,

$$
\sup _{n \geqslant 1} n^{p(\alpha-1 / 2)} \mathbb{P}\left\{\max _{1 \leqslant i \leqslant n}\left\|S_{i}\right\|>n^{\alpha} x\right\} \leqslant C(p, B) \sup _{t>0} t^{p} \mathbb{P}\left\{\left\|X_{1}\right\|>t\right\} x^{-p / 2-1} ;
$$

(2) if $X_{1} \in \mathbb{L}_{0}^{p, \infty}$ then for each $x>0$,

$$
\lim _{n \rightarrow+\infty} n^{p(\alpha-1 / 2)} \mathbb{P}\left\{\max _{1 \leqslant i \leqslant n}\left\|S_{i}\right\|>n^{\alpha} x\right\}=0 ;
$$

(3) if $X_{1} \in \mathbb{L}^{p}$ then

$$
\sum_{n=1}^{+\infty} n^{p(\alpha-1 / 2)-1} \mathbb{P}\left\{\max _{1 \leqslant i \leqslant n}\left\|S_{i}\right\|>n^{\alpha} x\right\} \leqslant C(p, B) x^{-p}\left\|X_{1}\right\|_{p}^{p} .
$$

For condition (C.2) to be satisfied, we require $X_{1} \in \mathbb{L}^{2} \log \mathbb{L}$ rather than in $\mathbb{L}^{2}$. One may wonder whether that stronger condition is really needed. Its "necessity" for stationary martingale differences sequences is proved below. It also show that the result of Theorems 2.3 and 2.4 do not hold for $p=2$.

Recall that $(X, \Sigma, \mathbb{P}, \theta)$ is a dynamical system if $(X, \Sigma, \mathbb{P})$ is a probability space and $\theta: X \rightarrow X$ is a measurable map such that $\mathbb{P}\left(\theta^{-1} A\right)=\mathbb{P}(A)$ for all $A \in \Sigma$. If $f: \Omega \rightarrow \mathbb{R}$ is measurable, then the sequence $\left(f \circ \theta^{i}\right)_{i \geqslant 1}$ is strictly stationary.

We start with the following lemma.

Lemma 2.5. Let $\gamma>1$. There exist a dynamical system $(X, \Sigma, \mathbb{P}, \theta)$ and a non-negative measurable function $f \geqslant 0$ on $X$ such that, for every $0<\varepsilon \leqslant 1, \int_{X} f\left(\log ^{+}(f)\right)^{1-\varepsilon} d \mathbb{P}<\infty$ and

$$
\sum_{n \geqslant 0} 2^{n(\gamma-1)} \mathbb{P}\left\{\sum_{i=0}^{2^{n}-1} f \circ \theta^{i}>2^{n \gamma}\right\}=+\infty .
$$

Proposition 2.6. Let $\alpha>1 / 2$. There exists a stationary (and ergodic) sequence of martingale differences $\left(X_{i}\right)_{i \geqslant 1}$ such that for every $0<\varepsilon \leqslant 1, \mathbb{E}\left[X_{1}^{2}\left(\log ^{+}\left|X_{1}\right|\right)^{1-\varepsilon}\right]<\infty$ and the series $\sum_{n \geqslant 1} 2^{n(2 \alpha-1)} \mathbb{P}\left\{\left|\sum_{i=1}^{2^{n}} X_{i}\right|>2^{n \alpha}\right\}$ diverges.

In [HL14], Baum-Katz type estimates have been formulated for martingales differences arrays, extending the results in [Als90]. It has been extended to the Banach space valued setting in [Hao13]. However, it seems that our results cannot be compared with those of [HL14] because these ones require a control in of the $\mathbb{L}^{p}$-norm of $n^{-1} \sum_{i=1}^{n} \mathbb{E}\left[\left|X_{i}\right|^{\gamma} \mid \mathcal{F}_{i-1}\right]$. 
Remark 2.7. A similar statement holds when $n^{p(\alpha-1 / 2)-1} \mathbb{P}\left\{\max _{1 \leqslant i \leqslant n}\left\|S_{i}\right\|>n^{\alpha} x\right\}$ is replaced by $2^{n(p(\alpha-1 / 2))} \mathbb{P}\left\{\max _{1 \leqslant i \leqslant 2^{n}}\left\|S_{i}\right\|>2^{n \alpha} x\right\}$ in item (3) of Theorems 2.2 and 2.4 In view of Theorem 3.7 in [LV01] when $\alpha=1$, the weight $2^{n p / 2}$ is optimal for stationary ergodic martingale differences sequences in the following sense: if $\left(R_{n}\right)_{n \geqslant 1}$ is a sequence of real numbers with goes to infinity and $p>2$, then there exists a stationary martingale differences sequence $\left(X_{i}\right)_{i \geqslant 0}$ such that $X_{1}$ belongs to $\mathbb{L}^{p}$ but the sequence $\left(2^{n p / 2} R_{n} \mathbb{P}\left\{\max _{1 \leqslant i \leqslant 2^{n}}\left|S_{i}\right|>2^{n}\right\}\right)_{n \geqslant 1}$ does not converge to 0 .

2.2.2. Orthomartingale differences random fields. Let $\left(X_{\mathbf{i}}\right)_{\mathbf{i} \in \mathbb{Z}^{d}}$ be an i.i.d. real-valued random field. Theorem 4.1 in [Gut78] gives the equivalence between the following two assertions for $\alpha>1 / 2$ and $p \geqslant \max \{1 / \alpha, 1\}$ :

(1) $X_{\mathbf{1}}$ belongs to $\mathbb{L}^{p} \log ^{d-1} \mathbb{L}$;

(2) for each positive $\varepsilon$,

$$
\sum_{\mathbf{n} \in \mathbb{N}^{d}}|\mathbf{n}|^{p \alpha-2} \mathbb{P}\left\{\max _{\mathbf{1} \preccurlyeq \mathbf{i} \preccurlyeq \mathbf{n}}\left\|S_{\mathbf{i}}\right\|>\varepsilon|\mathbf{n}|^{\alpha}\right\}<+\infty .
$$

Deviation inequalities has been used in [KL11, Lag16] for the question of complete convergence of orthomartingale differences random fields.

Similar results as in Subsubsection 2.2.1 can be proved for some orthomartingale differences random fields.

Theorem 2.8. Let $B$ be a separable $r$-smooth Banach space. For each B-valued orthomartingale differences random field $\left(X_{\mathbf{i}}\right)_{\mathbf{i} \in \mathbb{Z}^{d}}$ satisfying (1.15) and such that $X_{\mathbf{1}} \in \mathbb{L}^{r} \log ^{d} \mathbb{L}$, for each positive $\varepsilon$ and each $\alpha \in(1 / r, 1]$,

$$
\sum_{\mathbf{n} \in \mathbb{N}^{d}}|\mathbf{n}|^{r \alpha-2} \mathbb{P}\left\{\max _{\mathbf{1} \preccurlyeq \mathbf{i} \preccurlyeq \mathbf{n}}\left\|S_{\mathbf{i}}\right\|>\varepsilon|\mathbf{n}|^{\alpha}\right\}<+\infty .
$$

Remark 2.9. One could also formulate the corresponding result where $r$ is replaced in (2.13) by $1<p<r$. But this could be established in a more general context than ours, namely, that of stochastically dominated orthomartingale differences random fields, by using truncation arguments like in [DM07].

Theorem 2.10. Let $B$ be a separable 2-smooth Banach space and $p>2$. For each B-valued orthomartingale differences random field $\left(X_{\mathbf{i}}\right)_{\mathbf{i} \in \mathbb{Z}^{d}}$ satisfying (1.15) and such that $X_{\mathbf{1}} \in \mathbb{L}^{p} \log ^{d-1} \mathbb{L}$, for each positive $\varepsilon$ and each $\alpha \in(1 / 2,1]$,

$$
\sum_{\mathbf{n} \in \mathbb{N}^{d}}|\mathbf{n}|^{p(\alpha-1 / 2)-1} \mathbb{P}\left\{\max _{1 \preccurlyeq \mathbf{i} \preccurlyeq \mathbf{n}}\left\|S_{\mathbf{i}}\right\|>\varepsilon|\mathbf{n}|^{\alpha}\right\}<+\infty .
$$

Remark 2.11. One hand the results in [Lag16], we do not require boundedness of the conditional moments. On the other hand, their result do not require that $\left(\left|X_{\mathbf{i}}\right|\right)_{\mathbf{i} \in \mathbb{Z}^{d}}$ is identically distributed hence the results are not directly comparable.

\section{Proofs}

\subsection{Proofs of Theorems 1.3 and 1.7 .}

Proof of Theorem 1.3. We first start by a distribution function inequality, which was first established in the real valued case and $r^{\prime}=2$ in [Bur73] (see also [Pis75], p. 24 for a proof). 
Lemma 3.1. Let $(B,\|\cdot\|)$ be an $r$-smooth Banach space for some $1<r \leqslant 2$ and let $1<r^{\prime} \leqslant r$. Then for any $\delta \in(0,1)$ and any $B$-valued martingale differences sequence $\left(X_{i}\right)_{i \geqslant 1}$ with respect to the filtration $\left(\mathcal{F}_{i}\right)_{i \geqslant 1}$, the following inequality holds for any $n \geqslant 1$ and $x>0$ :

$$
\begin{aligned}
\mathbb{P}\left\{\max _{1 \leqslant i \leqslant n}\left\|S_{i}\right\|>2 x\right\} \leqslant & C_{r^{\prime}, B}\left(\frac{\delta}{1-\delta}\right)^{r^{\prime}} \mathbb{P}\left\{\max _{1 \leqslant i \leqslant n}\left\|S_{i}\right\|>x\right\} \\
& +\mathbb{P}\left\{\max _{1 \leqslant i \leqslant n}\left\|X_{i}\right\|>\delta x\right\}+\mathbb{P}\left\{\left(\sum_{i=1}^{n} \mathbb{E}\left[\left\|X_{i}\right\|^{r^{\prime}} \mid \mathcal{F}_{i-1}\right]\right)^{1 / r^{\prime}}>\delta x\right\},
\end{aligned}
$$

where $C_{r^{\prime}, B}$ is defined by (1.6) and $S_{n}=\sum_{i=1}^{n} X_{i}$.

Proof. We assume that $n \geqslant 2$ since for $n=1$, the result is obvious. We define $A_{1}=B_{1}=C_{1}=\emptyset$ and for $2 \leqslant i \leqslant n$,

$$
\begin{gathered}
A_{i}:=\left\{\max _{1 \leqslant u \leqslant i-1}\left\|S_{u}\right\| \in(x, 2 x)\right\}, \\
B_{i}:=\left\{\max _{1 \leqslant u \leqslant i-1}\left\|X_{u}\right\| \leqslant \delta x\right\}, \\
C_{i}:=\left\{\sum_{u=1}^{i} \mathbb{E}\left[\left\|X_{i}\right\|^{r^{\prime}} \mid \mathcal{F}_{u-1}\right] \leqslant(\delta x)^{r^{\prime}}\right\} .
\end{gathered}
$$

We then introduce

$$
Y_{i}:=\mathbf{1}_{A_{i}} \mathbf{1}_{B_{i}} \mathbf{1}_{C_{i}} X_{i}, \quad 1 \leqslant i \leqslant n
$$

We show that the following inclusion holds:

$$
\begin{array}{r}
\left\{\max _{1 \leqslant i \leqslant n}\left\|S_{i}\right\|>2 x\right\} \cap\left\{\max _{1 \leqslant i \leqslant n}\left\|X_{i}\right\| \leqslant \delta x\right\} \cap\left\{\left(\sum_{i=1}^{n} \mathbb{E}\left[\left\|X_{i}\right\|^{r^{\prime}} \mid \mathcal{F}_{i-1}\right]\right)^{1 / r^{\prime}} \leqslant \delta x\right\} \\
\subset\left\{\left\|\sum_{i=1}^{n} Y_{i}\right\|>(1-\delta) x\right\} .
\end{array}
$$

Indeed, let $\omega$ be an element of the left hand side of (3.6). Then for any $i \in\{2, \ldots, n\}, \omega$ belongs to $B_{i} \cap C_{i}$. Consequently, $\sum_{i=1}^{n} Y_{i}(\omega)=\sum_{i=2}^{n} \mathbf{1}_{A_{i}} X_{i}(\omega)$. Let $I:=\left\{i \in\{2, \ldots, n\}: \omega \in A_{i}\right\}$. Let $M_{i}:=\max _{1 \leqslant u \leqslant i}\left\|S_{i}\right\|$. Note that $\left\|S_{1}(\omega)\right\|=\left\|X_{1}(\omega)\right\| \leqslant \delta x<x$, since $0<\delta<1$ hence $M_{1}<x$ and $M_{n}>2 x$. Since for any $i \in\{1, \ldots, n-1\}$ we have $\left\|X_{i}(\omega)\right\| \leqslant \delta x$, it follows that $0 \leqslant M_{i+1}-M_{i} \leqslant \delta x$. Consequently, $I$ is of the form $\left\{i, i_{0} \leqslant i \leqslant j_{0}\right\}$ for some integers $i_{0} \geqslant 2$ and $j_{0} \leqslant n$. Therefore,

$$
\left\|\sum_{i=1}^{n} Y_{i}(\omega)\right\|=\left\|\sum_{i=i_{0}}^{j_{0}} X_{i}(\omega)\right\| \geqslant\left\|\sum_{i=1}^{j_{0}} X_{i}(\omega)\right\|-\left\|\sum_{i=1}^{i_{0}-1} X_{i}(\omega)\right\|-\left\|X_{i_{0}}(\omega)\right\| .
$$

Now, (3.6) holds in view of the inequalities $\left\|\sum_{i=1}^{j_{0}} X_{i}(\omega)\right\|>2 x,\left\|\sum_{i=1}^{i_{0}-1} X_{i}(\omega)\right\| \leqslant x\left(\right.$ since $\left.\omega \in I_{i_{0}}\right)$ and $\left\|X_{i_{0}}(\omega)\right\| \leqslant \delta x$.

Taking the probabilities on both sides in (3.6), one gets

$$
\begin{aligned}
\mathbb{P}\left(\left\{\max _{1 \leqslant i \leqslant n}\left\|S_{i}\right\|>2 x\right\}\right. & \left.\cap\left\{\max _{1 \leqslant i \leqslant n}\left\|X_{i}\right\| \leqslant \delta x\right\} \cap\left\{\left(\sum_{i=1}^{n} \mathbb{E}\left[\left\|X_{i}\right\|^{r^{\prime}} \mid \mathcal{F}_{i-1}\right]\right)^{1 / r^{\prime}} \leqslant \delta x\right\}\right) \\
& \leqslant \mathbb{P}\left\{\left\|\sum_{i=1}^{n} Y_{i}\right\|^{r^{\prime}}>((1-\delta) x)^{r^{\prime}}\right\} \leqslant((1-\delta) x)^{r^{\prime}} \mathbb{E}\left[\left\|\sum_{i=1}^{n} Y_{i}\right\|^{r^{\prime}}\right] .
\end{aligned}
$$


Observe that $A_{i}, B_{i}$ and $C_{i}$ belong to $\mathcal{F}_{i-1}$, hence $\left(Y_{i}\right)_{i \geqslant 1}$ is a martingale differences sequence. The combination of (3.8) with (1.6) yields

$$
\begin{array}{r}
\mathbb{P}\left(\left\{\max _{1 \leqslant i \leqslant n}\left\|S_{i}\right\|>2 x\right\} \cap\left\{\max _{1 \leqslant i \leqslant n}\left\|X_{i}\right\| \leqslant \delta x\right\} \cap\left\{\left(\sum_{i=1}^{n} \mathbb{E}\left[\left\|X_{i}\right\|^{r^{\prime}} \mid \mathcal{F}_{i-1}\right]\right)^{1 / r^{\prime}} \leqslant \delta x\right\}\right) \\
\leqslant((1-\delta) x)^{-r^{\prime}} C_{r^{\prime}, B} \sum_{i=1}^{n} \mathbb{E}\left[\left\|Y_{i}\right\|^{r^{\prime}}\right] .
\end{array}
$$

Since for $i \geqslant 2$,

$$
\mathbb{E}\left[\left\|Y_{i}\right\|^{r^{\prime}}\right]=\mathbb{E}\left[\mathbb{E}\left[\left\|Y_{i}\right\|^{r^{\prime}}\right] \mid \mathcal{F}_{i-1}\right]=\mathbb{E}\left[\mathbf{1}_{A_{i}} \mathbf{1}_{B_{i}} \mathbf{1}_{C_{i}} \mathbb{E}\left[\left\|X_{i}\right\|^{r^{\prime}} \mid \mathcal{F}_{i-1}\right]\right]
$$

we derive that

$$
\mathbb{E}\left[\left\|Y_{i}\right\|^{r^{\prime}}\right] \leqslant \mathbb{E}\left[\mathbf{1}\left\{\max _{1 \leqslant i \leqslant n}\left\|S_{i}\right\|>x\right\} \mathbf{1}_{C_{i}} \mathbb{E}\left[\left\|X_{i}\right\|^{r^{\prime}} \mid \mathcal{F}_{i-1}\right]\right]
$$

Observe that

$$
\sum_{i=2}^{n} \mathbf{1}_{C_{i}} \mathbb{E}\left[\left\|X_{i}\right\|^{r^{\prime}} \mid \mathcal{F}_{i-1}\right] \leqslant(\delta x)^{r^{\prime}}
$$

Indeed, if $Z_{i}$ are non-negative random variables, $Z_{i}^{\prime}:=\sum_{u=1}^{i} Z_{u}$ and $E_{i}=\left\{Z_{u}^{\prime} \leqslant t\right\}$, we have

$$
\begin{aligned}
\sum_{i=2}^{n} Z_{i} \mathbf{1}_{E_{i}} & =\sum_{i=2}^{n}\left(Z_{i}^{\prime}-Z_{i-1}^{\prime}\right) \mathbf{1}_{E_{i}} \\
& =\sum_{j=2}^{n} Z_{j}^{\prime} \mathbf{1}_{E_{j}}-\sum_{j=1}^{n-1} Z_{j}^{\prime} \mathbf{1}_{E_{j+1}} \\
& =Z_{n}^{\prime} \mathbf{1}_{E_{n}}+\sum_{j=2}^{n-1} Z_{j}^{\prime}\left(\mathbf{1}_{E_{j}}-\mathbf{1}_{E_{j+1}}\right)-Z_{1}^{\prime} \mathbf{1}_{E_{2}}
\end{aligned}
$$

since $E_{j+1} \subset E_{j}$, the second term is smaller than $\sum_{j=2}^{n-1} t\left(\mathbf{1}_{E_{j}}-\mathbf{1}_{E_{j+1}}\right)=t \mathbf{1}_{E_{2}}-t \mathbf{1}_{E_{n}}$ and consequently,

$$
\sum_{i=2}^{n} Z_{i} \mathbf{1}_{E_{i}} \leqslant\left(Z_{n}^{\prime}-t\right) \mathbf{1}_{E_{n}}+t \mathbf{1}_{E_{2}}-Z_{1}^{\prime} \mathbf{1}_{E_{2}} \leqslant t
$$

Combining (3.9), (3.11) and (3.12), we get (3.1). This ends the proof of Lemma 3.1.

Let us define the functions

$$
\begin{gathered}
f: x \mapsto \mathbb{P}\left\{\max _{1 \leqslant i \leqslant n}\left\|S_{i}\right\|>x\right\} \text { and } \\
g: x \mapsto \mathbb{P}\left\{\max _{1 \leqslant i \leqslant n}\left\|X_{i}\right\|>x\right\}+\mathbb{P}\left\{\left(\sum_{i=1}^{n} \mathbb{E}\left[\left\|X_{i}\right\|^{r^{\prime}} \mid \mathcal{F}_{i-1}\right]\right)^{1 / r^{\prime}}>x\right\} .
\end{gathered}
$$

We established in Lemma 3.1 that for any $x>0$ and any $\delta \in(0,1)$,

$$
f(2 x) \leqslant C_{r^{\prime}, B}\left(\frac{\delta}{1-\delta}\right)^{r^{\prime}} f(x)+g(\delta x) .
$$

Let $q>0$ be fixed and $\eta:=C_{r^{\prime}, B}\left(\frac{\delta}{1-\delta}\right)^{r^{\prime}}$. Let $t>0$ be fixed, $a_{n}:=f\left(2^{n} t\right), b_{n}:=\eta^{-n} a_{n}$ and $c_{n}:=g\left(2^{n} t \delta\right)$. Then

$$
b_{n+1}=\eta^{-n-1} a_{n+1} \leqslant \eta^{-n-1}\left(\eta a_{n}+c_{n}\right)=b_{n}+\eta^{-n-1} c_{n} .
$$


Consequently,

$$
b_{N}=b_{0}+\sum_{n=0}^{N-1} b_{n+1}-b_{n} \leqslant a_{0}+\sum_{n=0}^{N-1} \eta^{-n-1} c_{n},
$$

which gives

$$
a_{N} \leqslant a_{0} \eta^{N}+\sum_{n=0}^{N-1} \eta^{N-n-1} c_{n},
$$

and with the change of index $j=N-n$, we derive that for any positive $t$ and any integer $N$,

$$
f\left(2^{N} t\right) \leqslant f(t) \eta^{N}+\sum_{j=1}^{N} \eta^{j-1} g\left(\delta 2^{N-j} t\right) .
$$

Now, we choose $\delta:=2^{-1-q / r^{\prime}} C_{r^{\prime}, B}^{-1 / r^{\prime}}$, which is smaller than 1 , as $C_{r^{\prime}, B}$ is bigger than 1 . Applying (3.20) with $x=2^{N} t$ and letting $N$ going to infinity (accounting $f\left(2^{-N} x\right) \leqslant 1$ and $0<\eta<1$ ), we get

$$
f(x) \leqslant \sum_{j=1}^{+\infty} \eta^{j-1} g\left(\delta 2^{-j} x\right) .
$$

Since the function $g$ is non-increasing, we have

$$
\int_{2^{-j}}^{2^{-j+1}} g(u x \delta) u^{q-1} \mathrm{~d} u \geqslant g\left(2^{-j} x \delta\right) \int_{2^{-j}}^{2^{-j+1}} u^{q-1} \mathrm{~d} u=g\left(2^{-j} x \delta\right) \frac{2^{q}-1}{q} 2^{-j q}
$$

hence

$$
f(x) \leqslant \frac{q}{2^{q}-1} \sum_{j=1}^{+\infty} \eta^{j-1} 2^{j q} \int_{2^{-j}}^{2^{-j+1}} g(u x \delta) u^{q-1} \mathrm{~d} u .
$$

Notice that

$$
\eta \leqslant C_{r^{\prime}, B} 2^{r^{\prime}} 2^{-r^{\prime}-q} C_{r^{\prime}, B}^{-1} \leqslant 2^{-q},
$$

hence

$$
f(x) \leqslant \frac{q}{2^{q}-1} \eta^{-1} \int_{0}^{1} g(u x \delta) u^{q-1} \mathrm{~d} u .
$$

Since

$$
\eta^{-1}=\left(\frac{1-\delta}{\delta}\right)^{r^{\prime}} C_{r^{\prime}, B}^{-1} \leqslant\left(\frac{1}{\delta}\right)^{r^{\prime}} C_{r^{\prime}, B}^{-1} \leqslant 2^{q-r^{\prime}},
$$

we get (1.7). This ends the proof of Theorem 1.3.

Proof of Theorem 1.\%. We shall need the following lemma.

Lemma 3.2. Assume that $X$ and $Y$ are two non-negative random variables such that for each positive $x$, we have

$$
x \mathbb{P}\{X>x\} \leqslant \mathbb{E}[Y 1\{X \geqslant x\}] .
$$

Then for each $t$, the following inequality holds:

$$
\mathbb{P}\{X>2 t\} \leqslant \int_{1}^{+\infty} \mathbb{P}\{Y>s t\} \mathrm{d} s .
$$

Proof of Lemma 3.2. Rewriting the expectation as

$$
\mathbb{E}[Y \mathbf{1}\{X \geqslant 2 t\}]=\int_{0}^{+\infty} \mathbb{P}\{Y \mathbf{1}\{X \geqslant 2 t\}>u\} \mathrm{d} u \leqslant t \mathbb{P}\{X \geqslant 2 t\}+\int_{t}^{+\infty} \mathbb{P}\{Y>u\} \mathrm{d} u,
$$

we derive by the assumption the bound

$$
2 t \mathbb{P}\{X>2 t\} \leqslant t \mathbb{P}\{X \geqslant 2 t\}+\int_{t}^{+\infty} \mathbb{P}\{Y>u\} \mathrm{d} u .
$$


We conclude using the substitution $t s:=u$.

We apply Theorem 1.3. The first term of (1.7) is controlled in the following way, using the fact that if $U$ has uniform distribution on $[0,1]$, then $Q_{\left\|X_{i}\right\|}(U)$ has the same distribution as $\left\|X_{i}\right\|$ :

$$
\begin{aligned}
& \int_{0}^{1} \mathbb{P}\left\{\max _{1 \leqslant i \leqslant n}\left\|X_{i}\right\|>x u n^{1 / r^{\prime}}\right\} u^{q-1} \mathrm{~d} u \leqslant \sum_{i=1}^{n} \int_{0}^{1} \mathbb{P}\left\{\left\|X_{i}\right\|>x u n^{1 / r^{\prime}}\right\} u^{q-1} \mathrm{~d} u \\
& =\sum_{i=1}^{n} \int_{0}^{1} \lambda\left\{t \in[0,1], Q_{\left\|X_{i}\right\|}(t)>x_{u n}{ }^{1 / r^{\prime}}\right\} u^{q-1} \mathrm{~d} u \\
& \leqslant \sum_{i=1}^{n} \int_{0}^{1} \lambda\left\{t \in[0,1], Q_{X}(t)>B_{r^{\prime}, q} x u n^{1 / r^{\prime}}\right\} u^{q-1} \mathrm{~d} u \\
& =n \int_{0}^{1} \mathbb{P}\left\{X>\text { xun }^{1 / r^{\prime}}\right\} u^{q-1} \mathrm{~d} u,
\end{aligned}
$$

where $\lambda$ denotes the Lebesgue measure.

In order to control the second term of (1.7), we first bound $\sum_{i=1}^{n} \mathbb{E}\left[\left\|X_{i}\right\|^{r^{\prime}} \mid \mathcal{F}_{i-1}\right]$ by $\sum_{i=1}^{n} V_{i}$ and we notice that for any convex function $\phi: \mathbb{R} \rightarrow \mathbb{R}$,

$$
\mathbb{E}\left[\phi\left(\frac{1}{n} \sum_{i=1}^{n} V_{i}\right)\right] \leqslant \frac{1}{n} \sum_{i=1}^{n} \mathbb{E}\left[\phi\left(V_{i}\right)\right]=\mathbb{E}\left[\phi\left(V_{1}\right)\right] .
$$

By Theorem 6 in [Rue81], there exists a probability space $\left(\Omega^{\prime}, \mathcal{A}^{\prime}, \mathbb{P}^{\prime}\right)$ and random variables $Z_{n}^{\prime}$ and $Z^{\prime}$ such that $V_{n}^{\prime}$ has the same distribution as $\frac{1}{n} \sum_{i=1}^{n} V_{i}, Z^{\prime}$ has the same distribution as $V_{1}$ and such that $Z_{n}^{\prime}=\mathbb{E}\left[Z^{\prime} \mid Z_{n}^{\prime}\right]$.

Therefore, inequality (3.29) holds with $X:=Z_{n}^{\prime}$ and $Y=Z^{\prime}$, hence by Lemma 3.2 the estimate

$$
\mathbb{P}\left\{\frac{1}{n} \sum_{i=1}^{n} \mathbb{E}\left[\left\|X_{i}\right\|^{r^{\prime}} \mid \mathcal{F}_{i-1}\right]>2 u^{r^{\prime}} x^{r^{\prime}}\right\} \leqslant \int_{1}^{+\infty} \mathbb{P}\left\{V_{1}>u^{r^{\prime}} x^{r^{\prime}} s\right\} \mathrm{d} s
$$

is valid for any $n$. We can deduce from inequalities (1.7) and (3.34) that (1.10) is satisfied after having used the elementary identity

$$
\int_{0}^{1} \int_{1}^{+\infty} h\left(u^{r^{\prime}} v\right) \mathrm{d} v u^{q-1} \mathrm{~d} u=\frac{1}{q-r^{\prime}} \int_{0}^{+\infty} h(w) \min \left\{w^{\frac{q-r^{\prime}}{r^{\prime}}}, 1\right\} \mathrm{d} w
$$

with $h(t):=\mathbb{P}\left\{V_{1}>x^{r^{\prime}} t / 2\right\}$.

In order to prove (1.11), we bound the two terms of the right hand side of (1.7) independently of $n$. Let us start by the first term, which can be written as

$$
n^{-q / r^{\prime}} \int_{0}^{n^{1 / r^{\prime}}} \mathbb{P}\left\{\max _{1 \leqslant i \leqslant n}\left\|X_{i}\right\|>2^{-1-q / r^{\prime}} C_{r^{\prime}, B}^{-1 / r^{\prime}} x v\right\} v^{q-1} \mathrm{~d} v .
$$

If $v \leqslant 1$, we use the bound $n^{1-q / r^{\prime}} v^{q-1} \leqslant v^{q-1}$ (since $q>r^{\prime}$ ). If $1<v \leqslant n^{1 / r^{\prime}}$, then $n^{1-q / r^{\prime}} v^{q-1} \leqslant$ $v^{r^{\prime}-1}$. We thus have

$$
\begin{aligned}
\int_{0}^{1} \mathbb{P}\left\{\max _{1 \leqslant i \leqslant n}\left\|X_{i}\right\|>2^{-1-q / r^{\prime}}\right. & \left.C_{r^{\prime}, B}^{-1 / r^{\prime}} n^{1 / r^{\prime}} x u\right\} u^{q-1} \mathrm{~d} u \\
& \leqslant \int_{0}^{+\infty} \mathbb{P}\left\{\left\|X_{1}\right\|>2^{-1-q / r^{\prime}} C_{r^{\prime}, B}^{-1 / r^{\prime}} x v\right\} \min \left\{v^{q-1}, v^{r^{\prime}-1}\right\} \mathrm{d} v .
\end{aligned}
$$


Let us treat the second term. For any convex function $\phi: \mathbb{R} \rightarrow \mathbb{R}$,

$$
\begin{aligned}
\mathbb{E}\left[\phi\left(\frac{1}{n} \sum_{i=1}^{n} \mathbb{E}\left[\left\|X_{i}\right\|^{r^{\prime}} \mid \mathcal{F}_{i-1}\right]\right)\right] & \leqslant \frac{1}{n} \sum_{i=1}^{n} \mathbb{E}\left[\phi\left(\mathbb{E}\left[\left\|X_{i}\right\|^{r^{\prime}} \mid \mathcal{F}_{i-1}\right]\right)\right] \\
& \leqslant \frac{1}{n} \sum_{i=1}^{n} \mathbb{E}\left[\mathbb{E}\left[\phi\left(\left\|X_{i}\right\|^{r^{\prime}}\right) \mid \mathcal{F}_{i-1}\right]\right]=\mathbb{E}\left[\phi\left(\left\|X_{1}\right\|^{r^{\prime}}\right)\right] .
\end{aligned}
$$

Using again Theorem 6 in [Rue81], we derive that

$$
\begin{aligned}
\int_{0}^{1} \mathbb{P}\left\{\left(\sum_{i=1}^{n} \mathbb{E}\left[\left\|X_{i}\right\|^{r^{\prime}} \mid \mathcal{F}_{i-1}\right]\right)^{1 / r^{\prime}}\right. & \left.>2^{-1-q / r^{\prime}} C_{r^{\prime}, B}^{-1 / r^{\prime}} x u\right\} u^{q-1} \mathrm{~d} u \\
& \leqslant \int_{0}^{1} \int_{1}^{+\infty} \mathbb{P}\left\{\left\|X_{1}\right\|>2^{-1-q / r^{\prime}} C_{r^{\prime}, B}^{-1 / r^{\prime}} x u\right\} u^{q-1} \mathrm{~d} u
\end{aligned}
$$

Combining (3.37) and (3.38), we get (1.11). This ends the proof of Theorem 1.7.

3.2. Proof of Theorem 1.13. Let us prove (1.16). Let $B$ be a separable $r$-smooth Banach space and let $r^{\prime} \in(1,2], q>r^{\prime}$ be fixed.

Lemma 3.3. Let $\left(f_{d}\right)_{d \geqslant 1}$ be a sequence of functions from $(0,+\infty)$ to ifself such that:

(1) for any martingale differences sequence $\left(X_{i}\right)_{i \geqslant 1}$ with values in $B$ such that $\left(\left\|X_{i}\right\|\right)_{i \geqslant 1}$ is identically distributed and $\mathbb{E}\left[\left\|X_{1}\right\|^{r^{\prime}}\right]<+\infty$, any $n \geqslant 1$ and any positive $x$,

$$
\mathbb{P}\left\{\max _{1 \leqslant i \leqslant n}\left\|S_{i}\right\|>n^{1 / r^{\prime}} x\right\} \leqslant \int_{0}^{+\infty} \mathbb{P}\left\{\left\|X_{1}\right\|>x v\right\} f_{1}(v) \mathrm{d} v,
$$

where $S_{n}=\sum_{i=1}^{n} X_{i}$

(2) for any $d \geqslant 2$ and any positive $w$,

$$
f_{d}(w) \geqslant \int_{0}^{+\infty} \int_{0}^{+\infty} f_{d-1}(u) f_{1}\left(u^{\prime}\right) \frac{1}{u u^{\prime}} \mathbf{1}\left\{u u^{\prime} \leqslant w\right\} \mathrm{d} u \mathrm{~d} u^{\prime} .
$$

Then for any integer $d \geqslant 1$, any orthomartingale differences random field $\left(X_{\mathbf{i}}\right)_{\mathbf{i} \in \mathbb{Z}^{d}}$ satisfying (1.15), $\mathbb{E}\left[\left\|X_{1}\right\|^{r^{\prime}}\right]<+\infty$, any $\mathbf{n} \succcurlyeq \mathbf{1}$ and any positive $x$

$$
\mathbb{P}\left\{\max _{\mathbf{1} \preccurlyeq \mathbf{i} \preccurlyeq \mathbf{n}}\left\|S_{\mathbf{i}}\right\|>2^{d-1} x|\mathbf{n}|^{1 / r^{\prime}}\right\} \leqslant \int_{0}^{+\infty} \mathbb{P}\left\{\left\|X_{\mathbf{1}}\right\|>x v\right\} f_{d}(v) \mathrm{d} v,
$$

where $S_{\mathbf{i}}$ is defined by (1.14).

For $p>0$ and $k \in \mathbb{N}$, let

$$
a_{p, k}:=\int_{0}^{1} t^{p-1}(1+|\log t|)^{k} \mathrm{dt} .
$$

Lemma 3.4. Let $\left(c_{d}\right)_{d \geqslant 1}$ be the sequence of real numbers such that

$$
\begin{gathered}
c_{1}=\frac{2^{q+1}}{2^{q}-1} \frac{q 2^{-r^{\prime}}}{q-r^{\prime}} \text { and } \\
c_{d}=c_{d-1}\left(1+a_{q-r^{\prime}, d-2}+2^{d-1} a_{q-r^{\prime}, d-2}\right) .
\end{gathered}
$$

Then the sequence of functions $\left(f_{d}\right)_{d \geqslant 1}$ defined by

$$
f_{d}: u \mapsto c_{d} \min \left\{u^{q-1}, u^{r^{\prime}-1}\right\}(1+|\log u|)^{d-1}, \quad u>0
$$

satisfies the conditions of Lemma 3.3. 
Inequality (1.16) is a direct consequence of Lemmas 3.3 and 3.4.

Proof of Lemma 3.3. The proof is done by induction on $d$. The case $d=1$ is contained in the assumptions. Assume that inequality (3.41) holds for some $d \geqslant 1$ for any orthomartingale differences random field $\left(X_{\mathbf{i}}\right)_{\mathbf{i} \in \mathbb{Z}^{d}}$ satisfying (1.15), $\mathbb{E}\left[\left\|X_{\mathbf{1}}\right\|^{r^{\prime}}\right]<+\infty$, any $\mathbf{n} \succcurlyeq \mathbf{1}$ and any positive $x$.

Let $\left(X_{\mathbf{i}}\right)_{\mathbf{i} \in \mathbb{Z}^{d+1}}$ be an orthomartingale differences random field with respect to the commutatitve filtration $\left(\mathcal{F}_{\mathbf{i}}\right)_{\mathbf{i} \in \mathbb{Z}^{d+1}}$ satisfying (1.15). Using property (P.2) in Lemma 1.12 and Lemma 3.2 applied to

$$
X=\max _{\substack{1 \leqslant i_{k} \leqslant n_{k} \\ 1 \leqslant k \leqslant d+1}}\left\|S_{\mathbf{i}}\right\| \text { and } Y=\max _{\substack{1 \leqslant i_{k} \leqslant n_{k} \\ 1 \leqslant k \leqslant d}}\left\|S_{\mathbf{i}, n_{d+1}}\right\|
$$

we get

$$
\mathbb{P}\left\{\max _{1 \preccurlyeq \mathbf{i} \preccurlyeq \mathbf{n}}\left\|S_{\mathbf{i}}\right\|>2^{d} x|\mathbf{n}|^{1 / r^{\prime}}\right\} \leqslant \int_{1}^{+\infty} \mathbb{P}\left\{\max _{\substack{1 \leqslant i_{k} \leqslant n_{k} \\ 1 \leqslant k \leqslant d}}\left\|S_{\mathbf{i}, n_{d+1}}\right\|>2^{d-1} x|\mathbf{n}|^{1 / r^{\prime}} v\right\} \mathrm{d} v .
$$

We now apply the induction hypothesis to $\widetilde{X_{\mathbf{i}}}:=\sum_{k=1}^{n_{d+1}} X_{\mathbf{i}, k}, \widetilde{\mathcal{F}}_{\mathbf{i}}:=\mathcal{F}_{\mathbf{i}, n_{d+1}}$ and $\widetilde{x}:=x n_{d+1}^{1 / r^{\prime}}$ to get

$$
\mathbb{P}\left\{\max _{\mathbf{1} \preccurlyeq \mathbf{i} \preccurlyeq \mathbf{n}}\left\|S_{\mathbf{i}}\right\|>2^{d} x|\mathbf{n}|^{1 / r^{\prime}}\right\} \leqslant \int_{1}^{+\infty} \int_{0}^{+\infty} \mathbb{P}\left\{\left\|\sum_{k=1}^{n_{d+1}} X_{1, k}\right\|>x n_{d+1}^{1 / r^{\prime}} v u\right\} f_{d-1}(u) \mathrm{d} u \mathrm{~d} v .
$$

After having appylied the one dimensional case, we derive that

$$
\mathbb{P}\left\{\max _{\mathbf{1} \preccurlyeq \mathbf{i} \preccurlyeq \mathbf{n}}\left\|S_{\mathbf{i}}\right\|>2^{d} x|\mathbf{n}|^{1 / r^{\prime}}\right\} \leqslant \int_{(0,+\infty)^{3}} \mathbb{P}\left\{\left\|X_{\mathbf{1}}\right\|>x v u u^{\prime}\right\} f_{d-1}(u) f_{1}\left(u^{\prime}\right) \mathbf{1}\{v>1\} \mathrm{d} v \mathrm{~d} u \mathrm{~d} u^{\prime} .
$$

and the substitution $w:=v u u^{\prime}$ for fixed $u$ and $u^{\prime}$ combined with (3.40) end the proof of Lemma 3.3.

Proof of Lemma 3.4. Item 1 follows from Theorem 1.7 after a substitution in the integral of the right hand side of (1.11).

Let us show item 2. Let $d \geqslant 2$ be fixed. Observe that

$$
\begin{aligned}
\int_{0}^{+\infty} f_{1}\left(u^{\prime}\right) \frac{1}{u^{\prime}} \mathbf{1}\left\{u u^{\prime} \leqslant w\right\} \mathrm{d} u^{\prime} & =c_{1} \int_{0}^{w / u} \frac{1}{v} \min \left\{v^{q-1}, v^{r^{\prime}-1}\right\} \mathrm{d} v \\
& \leqslant c_{1} \min \left\{\int_{0}^{w / u} v^{q-2} \mathrm{~d} v, \int_{0}^{w / u} v^{r^{\prime}-2} \mathrm{~d} v\right\} \\
& \leqslant \frac{c_{1}}{r^{\prime}-1} \min \left\{\left(\frac{w}{u}\right)^{q-1},\left(\frac{w}{u}\right)^{r^{\prime}-1}\right\},
\end{aligned}
$$

hence

$$
\begin{aligned}
& \int_{0}^{+\infty} \int_{0}^{+\infty} f_{d-1}(u) f_{1}\left(u^{\prime}\right) \frac{1}{u u^{\prime}} \mathbf{1}\left\{u u^{\prime} \leqslant w\right\} \mathrm{d} u \mathrm{~d} u^{\prime} \\
& \quad \leqslant \frac{c_{1}}{r^{\prime}-1} \int_{0}^{+\infty} f_{d-1}(u) \min \left\{\left(\frac{w}{u}\right)^{q-1},\left(\frac{w}{u}\right)^{r^{\prime}-1}\right\} \mathrm{d} u
\end{aligned}
$$

Let $g: u \mapsto f_{d-1}(u) u^{-1} \min \left\{\left(\frac{w}{u}\right)^{q-1},\left(\frac{w}{u}\right)^{r^{\prime}-1}\right\}$ and $I(w):=\int_{0}^{+\infty} g(u) \mathrm{d} u$. Assume that $w \leqslant 1$. Spliting the integral into three parts (from 0 to $w$, from $w$ to 1 and from 1 to infinity), we get

$$
\begin{aligned}
I(w) & =c_{d-1} \int_{0}^{w} u^{q-1}(1+|\log u|)^{d-2} u^{-1}\left(\frac{w}{u}\right)^{r^{\prime}-1} \mathrm{~d} u+c_{d-1} \int_{w}^{1} u^{q-1}(1+|\log u|)^{d-2} u^{-1}\left(\frac{w}{u}\right)^{q-1} \mathrm{~d} u \\
& +c_{d-1} \int_{1}^{+\infty} u^{r^{\prime}-1}(1+|\log u|)^{d-2} u^{-1}\left(\frac{w}{u}\right)^{q-1} \mathrm{~d} u=: c_{d-1}\left(I_{1}(w)+I_{2}(w)+I_{3}(w)\right) .
\end{aligned}
$$


Let us bound these integrals. We have

$$
I_{1}(w)=w^{r^{\prime}-1} \int_{0}^{w} u^{q-r^{\prime}-1}(1+|\log u|)^{d-2} \mathrm{~d} u
$$

and the substitution $x=u / w$ gives

$$
I_{1}(w)=w^{q-1} \int_{0}^{1} x^{q-r^{\prime}-1}(1+|\log x|+|\log w|)^{d-2} \mathrm{~d} x \leqslant w^{q-1} 2^{d-1} a_{q-r^{\prime}, d-2}(1+|\log w|)^{d-2} .
$$

Observe that for $u \in(w, 1)$,

$$
u^{q-1}(1+|\log u|)^{d-2} u^{-1}\left(\frac{w}{u}\right)^{q-1}=w^{q-1}(1+|\log u|)^{d-2} u^{-1} \leqslant w^{q-1}(1+|\log w|)^{d-2} u^{-1}
$$

hence

$$
I_{2}(w) \leqslant w^{q-1}(1+|\log w|)^{d-1} .
$$

Finally,

$$
I_{3}(w)=w^{q-1} \int_{1}^{+\infty} \frac{1}{u^{q-r^{\prime}+1}}(1+|\log u|)^{d-2}=a_{q-r^{\prime}, d-2} w^{q-1}
$$

hence

$$
I(w) \leqslant c_{d-1} w^{q-1}(1+|\log w|)^{d-1}\left(1+a_{q-r^{\prime}, d-2}+2^{d-1} a_{q-r^{\prime}, d-2}\right) .
$$

Now, if $w>1$, a similar result by spliting the integral into three parts (from 0 to $1 w$, from 1 to $w$ and from 1 to infinity) yields for $w>1$ :

$$
I(w) \leqslant c_{d-1} w^{r^{\prime}-1}(1+|\log w|)^{d-1}\left(1+a_{q-r^{\prime}, d-2}+2^{d-1} a_{q-r^{\prime}, d-2}\right) .
$$

This concludes the proof of Lemma 3.4.

\subsection{Proof of the results of Section 2.}

Proof of Theorem 2.1. A computation gives that

$$
\theta_{n}-\theta=\frac{\sum_{i=1}^{n} \phi_{i} \varepsilon_{i}}{\sum_{j=1}^{n} \phi_{j}^{2}}
$$

We define

$$
\begin{gathered}
\xi_{i}:=\frac{\phi_{i} \varepsilon_{i}}{\sum_{j=1}^{n} \phi_{j}^{2}} \\
\mathcal{F}_{i}:=\sigma\left(\varepsilon_{u}, 1 \leqslant u \leqslant i, \phi_{j}, 1 \leqslant j \leqslant n\right), \quad i \geqslant 1, \mathcal{F}_{0}=\sigma\left(\phi_{j}, 1 \leqslant j \leqslant n\right),
\end{gathered}
$$

and $\mathcal{G}_{i}:=\sigma\left(\varepsilon_{u}, 1 \leqslant u \leqslant i\right)$ for $i \geqslant 1$ and $\mathcal{G}_{0}=\{\emptyset, \Omega\}$. In this way, for $i \geqslant 2$,

$$
\mathbb{E}\left[\xi_{i} \mid \mathcal{F}_{i-1}\right]=\frac{\phi_{i}}{\sum_{j=1}^{n} \phi_{j}^{2}} \mathbb{E}\left[\varepsilon_{i} \mid \mathcal{F}_{i-1}\right]
$$

Since $\sigma\left(\phi_{j}, 1 \leqslant j \leqslant n\right)$ is independent of $\sigma\left(\varepsilon_{u}, 1 \leqslant u \leqslant i\right)$, equality

$$
\mathbb{E}\left[\varepsilon_{i} \mid \mathcal{F}_{i-1}\right]=\mathbb{E}\left[\varepsilon_{i} \mid \sigma\left(\varepsilon_{u}, 1 \leqslant u \leqslant i\right)\right]
$$

holds and the right hand side was assumed to be equal to zero. Moreover, by independence, $\mathbb{E}\left[\xi_{1} \mid \mathcal{F}_{0}\right]=$ 0 hence $\left(\xi_{i}, \mathcal{F}_{i}\right)_{i \geqslant 1}$ is a martingale differences sequence. Since $\left(\theta_{n}-\theta\right) \sqrt{\sum_{i=1}^{n} \phi_{i}^{2}}=\sum_{i=1}^{n} \xi_{i}$, an application of Theorem 1.3 with $B=\mathbb{R}$ and $r^{\prime}=2$ yields

$$
\mathbb{P}\left\{\left|\theta_{n}-\theta\right| \sqrt{\sum_{i=1}^{n} \phi_{i}^{2}}>x\right\} \leqslant A_{1}+A_{2},
$$

where

$$
A_{1}=\frac{2^{q-2}}{2^{q}-1} q \int_{0}^{1} \mathbb{P}\left\{\max _{1 \leqslant i \leqslant n}\left|\xi_{i}\right|>2^{-1-q / 2} x u\right\} u^{q-1} \mathrm{~d} u
$$




$$
A_{2}=\frac{2^{q-2}}{2^{q}-1} q \int_{0}^{1} \mathbb{P}\left\{\left(\sum_{i=1}^{n} \mathbb{E}\left[\xi_{i}^{2} \mid \mathcal{F}_{i-1}\right]\right)^{1 / 2}>2^{-1-q / 2} x u\right\} u^{q-1} \mathrm{~d} u .
$$

We bound $A_{1}$ using Markov's inequality:

$$
\begin{aligned}
A_{1} & \leqslant \frac{2^{q-2}}{2^{q}-1} q \sum_{i=1}^{n} \int_{0}^{1} \mathbb{P}\left\{\left|\xi_{i}\right|>2^{-1-q / 2} x u\right\} u^{q-1} \mathrm{~d} u \\
& \leqslant \frac{2^{q-2}}{2^{q}-1} \frac{q}{q-p} \sum_{i=1}^{n} \mathbb{E}\left[\left|\xi_{i}\right|^{p}\right] 2^{p+p q / 2} x^{-p} .
\end{aligned}
$$

Using independence and the convexity inequality $\sum_{i=1}^{n}\left|\phi_{i}\right|^{p} \leqslant\left(\sum_{i=1}^{n} \phi_{i}^{2}\right)^{p / 2}$ valid for $p \geqslant 2$, we get that $\mathbb{E}\left[\left|\xi_{i}\right|^{p}\right] \leqslant C_{1}$ hence

$$
A_{1} \leqslant C_{1} \frac{2^{q-2}}{2^{q}-1} \frac{q}{q-p} 2^{p+p q / 2} x^{-p}
$$

Now, in order to bound $A_{2}$, we notice that

$$
\mathbb{E}\left[\xi_{i}^{2} \mid \mathcal{F}_{i-1}\right]=\frac{\phi_{i}^{2}}{\sum_{j=1}^{n} \phi_{j}^{2}} \mathbb{E}\left[\varepsilon_{i}^{2} \mid \mathcal{F}_{i-1}\right],
$$

and since $\varepsilon_{i}^{2}$ is independent of $\left(\phi_{j}, 1 \leqslant j \leqslant n\right)$, we derive that

$$
\mathbb{E}\left[\xi_{i}^{2} \mid \mathcal{F}_{i-1}\right]=\frac{\phi_{i}^{2}}{\sum_{j=1}^{n} \phi_{j}^{2}} \mathbb{E}\left[\varepsilon_{i}^{2} \mid \mathcal{G}_{i-1}\right] \leqslant C_{2} \frac{\phi_{i}^{2}}{\sum_{j=1}^{n} \phi_{j}^{2}} .
$$

Consequently,

$$
\left(\sum_{i=1}^{n} \mathbb{E}\left[\xi_{i}^{2} \mid \mathcal{F}_{i-1}\right]\right)^{1 / 2} \leqslant \sqrt{C_{2}}
$$

and

$$
A_{2} \leqslant \frac{2^{q-2}}{2^{q}-1} q 2^{q+q^{2} / 2} x^{-q} C_{2}^{q / 2} .
$$

Theorem 2.1 follows from the combination of (3.62), (3.67) and (3.71).

Proof of Theorem 2.2. We use inequality (1.11) with $r^{\prime}=r$ and $q=2 r$ to get that for some constants $C$ and $c$ depending only on $r$ and $B$,

$$
\begin{aligned}
\mathbb{P}\left\{\max _{1 \leqslant i \leqslant n}\left\|S_{i}\right\|>n^{\alpha-1 / r} n^{1 / r} x\right\} \leqslant & C n \int_{0}^{1} \mathbb{P}\left\{X>c n^{\alpha} x u\right\} u^{2 r-1} \mathrm{~d} u \\
& +C \int_{0}^{+\infty} \mathbb{P}\left\{V_{1}^{1 / r}>c n^{\alpha-1 / r} x u\right\} \min \left\{u^{2 r-1}, u^{r-1}\right\} \mathrm{d} u .
\end{aligned}
$$

Observe that

$$
\begin{aligned}
\sum_{n=1}^{+\infty} n^{r \alpha-1} \mathbb{P}\left\{X>c n^{\alpha} x u\right\} & =\sum_{n=1}^{+\infty} n^{r \alpha-1} \sum_{k=n}^{+\infty} \mathbb{P}\left\{X \in\left(k^{\alpha} x u,(k+1)^{\alpha} x u\right]\right\} \\
& =\sum_{k=1}^{+\infty} \sum_{n=1}^{k} n^{r \alpha-1} \mathbb{P}\left\{X \in\left(k^{\alpha} x u,(k+1)^{\alpha} x u\right]\right\} \\
& \leqslant \sum_{k=1}^{+\infty} k^{r \alpha} \mathbb{P}\left\{X \in\left(k^{\alpha} x u,(k+1)^{\alpha} x u\right]\right\} \\
& \leqslant(x u)^{-r} \mathbb{E}\left[X^{r}\right]
\end{aligned}
$$


hence

$$
C \sum_{n=1}^{+\infty} n^{r \alpha-2} n \int_{0}^{1} \mathbb{P}\left\{X>c n^{\alpha} x u\right\} u^{2 r-1} \mathrm{~d} u \leqslant C c^{-r} x^{-r} \int_{0}^{1} u^{r-1} \mathrm{~d} u .
$$

Since for any non-negative random variable $Y, \sum_{n=1}^{+\infty} n^{r \alpha-2} \mathbb{P}\left\{Y>n^{\alpha-1 / r}\right\} \leqslant \mathbb{E}[Y \mathbf{1}\{Y \geqslant 1\}]$, we have

$$
\sum_{n=1}^{+\infty} n^{r \alpha-2} \mathbb{P}\left\{V_{1}^{1 / r}>c n^{\alpha-1 / r} x u\right\} \leqslant(c u x)^{-r} \mathbb{E}\left[V_{1} \mathbf{1}\left\{V_{1}^{1 / r} \geqslant c x u\right\}\right]
$$

which implies

$$
\begin{aligned}
\sum_{n=1}^{+\infty} n^{r \alpha-2} C \int_{0}^{+\infty} \mathbb{P}\left\{V_{1}^{1 / r}>\right. & \left.c n^{\alpha-1 / r} x u\right\} \min \left\{u^{2 r-1}, u^{r-1}\right\} \mathrm{d} u \\
& \leqslant C(c x)^{-r} \int_{0}^{+\infty} \mathbb{E}\left[V_{1} \mathbf{1}\left\{V_{1}^{1 / r} \geqslant c x u\right\}\right] \min \left\{u^{r-1}, u^{-1}\right\} \mathrm{d} u .
\end{aligned}
$$

Now for any non-negative real number $y$, let $h(y):=\int_{0}^{y} \min \left\{u^{r-1}, u^{-1}\right\} \mathrm{d} u$. If $y \leqslant 1$, then $h(y)=$ $y^{r} / r$ and if $y>1$, then

$$
h(y)=\int_{0}^{1} u^{r-1} \mathrm{~d} u+\int_{1}^{y} u^{-1} \mathrm{~d} u=\frac{1}{r}+\log y
$$

Since

$\int_{0}^{+\infty} \mathbb{E}\left[V_{1} \mathbf{1}\left\{V_{1}^{1 / r} \geqslant c x u\right\}\right] \min \left\{u^{r-1}, u^{-1}\right\} \mathrm{d} u=\mathbb{E}\left[V_{1} h\left(\frac{V_{1}^{1 / r}}{c x}\right)\right] \leqslant \frac{1}{r} \mathbb{E}\left[V_{1}\right]+\frac{1}{r} \mathbb{E}\left[V_{1} \log ^{+}\left(V_{1}\right)\right]$, we get the convergence of the series $\sum_{n=1}^{+\infty} n^{r \alpha-2} \mathbb{P}\left\{\max _{1 \leqslant i \leqslant n}\left\|S_{i}\right\|>n^{\alpha} x\right\}$.

Proof of Theorem 2.3. We use inequality (1.10) with $\widetilde{x}:=x 2^{n(\alpha-1 / 2)}, r^{\prime}=2$ and $q=2 p$. We get

$$
\begin{aligned}
n^{p(\alpha-1 / 2)} \mathbb{P}\left\{\max _{1 \leqslant i \leqslant n}\left\|S_{i}\right\|>n^{\alpha} x\right\} & \leqslant C n^{p(\alpha-1 / 2)+1} \int_{0}^{1} \mathbb{P}\left\{X>c x u n^{\alpha}\right\} u^{2 p-1} \mathrm{~d} u \\
& +C n^{p(\alpha-1 / 2)} \int_{0}^{+\infty} \mathbb{P}\left\{V_{1}>x^{2} u^{2} n^{2 \alpha-1}\right\} \min \left\{u^{2 p-1}, u\right\} \mathrm{d} u .
\end{aligned}
$$

(1) Assume that $X_{1}$ belongs to $\mathbb{L}^{p / 2+1, \infty}$ and $V_{1} \in \mathbb{L}^{p / 2, \infty}$. One bounds the first term of the right hand side of (3.77) by

$$
\begin{aligned}
C n^{p(\alpha-1 / 2)+1} \sup _{t>0} t^{p / 2+1} \mathbb{P}\{X>t\} \int_{0}^{1}\left(\text { cxun }^{\alpha}\right)^{-(p / 2+1)} u^{2 p-1} \mathrm{~d} u \\
=n^{\left(\frac{p}{2}-1\right)(\alpha-1)} C \sup _{t>0} t^{p / 2+1} \mathbb{P}\{X>t\}(c x)^{-(p / 2+1)} \int_{0}^{1} u^{3 p / 2-2} \mathrm{~d} u
\end{aligned}
$$

and use $\left(\frac{p}{2}-1\right)(\alpha-1) \leqslant 0$. One bounds the second term of the right hand side of (3.77) by

$$
\begin{aligned}
C n^{p(\alpha-1 / 2)} \sup _{t>0} t^{p / 2} \mathbb{P}\left\{V_{1}>t\right\} \int_{0}^{+\infty} & \left(c x^{2} u^{2} n^{2 \alpha-1}\right)^{-p / 2} \min \left\{u^{2 p-1}, u\right\} \mathrm{d} u \\
= & C(c x)^{-p} \sup _{t>0} t^{p / 2} \mathbb{P}\left\{V_{1}>t\right\} \int_{0}^{+\infty} \min \left\{u^{p-1}, u^{1-p}\right\} \mathrm{d} u,
\end{aligned}
$$

and since $p>2$, the latter integral is finite. 
(2) Assume that $X_{1} \in \mathbb{L}_{0}^{p / 2+1, \infty}$ and $V_{1} \in \mathbb{L}^{p / 2, \infty}$. Plugging the bounds

$$
\begin{gathered}
\mathbb{P}\left\{X>\text { cxun }^{\alpha}\right\} \leqslant\left(\text { cxun }^{\alpha}\right)^{-p / 2-1} \sup _{t>c x u 2^{n}} t^{p / 2+1} \mathbb{P}\{X>t\} \text { and } \\
\mathbb{P}\left\{V_{1}>c x^{2} u^{2} n^{2 \alpha-1}\right\} \leqslant\left(c x^{2} u^{2} n^{2 \alpha-1}\right)^{-p / 2} \sup _{t>c x^{2} u^{2} n^{2 \alpha-1}} t^{p / 2} \mathbb{P}\left\{V_{1}>t\right\} .
\end{gathered}
$$

into (3.77), we get

$$
\begin{aligned}
n^{p(\alpha-1 / 2)} \mathbb{P}\left\{\max _{1 \leqslant i \leqslant n}\left\|S_{i}\right\|\right. & \left.>n^{\alpha} x\right\} \leqslant C(c x)^{-p / 2-1} \int_{0}^{1} \sup _{t>c x u n^{\alpha}} t^{p / 2+1} \mathbb{P}\{X>t\} u^{3 s / 2-2} \mathrm{~d} u \\
& +C\left(c x^{2}\right)^{-p / 2} \int_{0}^{+\infty} \sup _{t>c x^{2} u^{2} n^{\alpha}} t^{p / 2} \mathbb{P}\left\{V_{1}>t\right\} \max \left\{u^{p-1}, u^{1-p}\right\} \mathrm{d} u
\end{aligned}
$$

and the right hand side goes to zero by monotone convergence.

(3) Assume that $X \in \mathbb{L}^{p / 2+1}$ and $V_{1} \in \mathbb{L}^{p / 2}$. In view of (3.77), we have

$$
\begin{aligned}
& \sum_{n=1}^{+\infty} n^{p(\alpha-1 / 2)} \mathbb{P}\left\{\max _{1 \leqslant i \leqslant n} \|\right.\left.S_{i}(m) \|>n^{\alpha} x\right\} \leqslant C \sum_{n=1}^{+\infty} n^{p(\alpha-1 / 2)+1} \int_{0}^{1} \mathbb{P}\left\{X>c x u n^{\alpha}\right\} u^{2 p-1} \mathrm{~d} u \\
&+C \sum_{n=1}^{+\infty} n^{p(\alpha-1 / 2)} \int_{0}^{+\infty} \mathbb{P}\left\{V_{1}>c x^{2} u^{2} n^{\alpha-1 / 2}\right\} \min \left\{u^{2 p-1}, u\right\} \mathrm{d} u
\end{aligned}
$$

Since for any non-negative random variable $Y$ and any $q>2, \sum_{n=1}^{+\infty} n^{q-1} \mathbb{P}\{Y>n\} \leqslant \mathbb{E}\left[Y^{q}\right]$, we get the conclusion of item 3 of Theorem 2.3 .

The proof of Theorem 2.4 is completely analogous hence omitted.

Proof of Lemma 2.5. We use the skyscrapers construction of Kakutani as in [BK65].

Let $\left(\ell_{n}\right)_{n \geqslant 1}$ be a non-increasing sequence of non-negative real numbers such that $\sum_{n \geqslant 1} \ell_{n}=1$. For every integer $n \geqslant 1$, set $X_{n}:=\left[0, \ell_{n}\right] \times\{n\}\left(\left[0, \ell_{n}\right]\right.$ equipped with the Lebesgue measure). Define then $X:=\cup_{n \geqslant 1} X_{n}$. Let $\tau$ be an ergodic transformation of $\left[0, \ell_{0}\right]$. Define an ergodic transformation $\theta$ on $X$ by $\theta(x, n)=(x, n+1)$ if $(x, n+1) \in X$ and by $\theta(x, n)=(\tau(x), 0)$ otherwise.

Let $n \geqslant 0$. For every $2^{n} \leqslant k \leqslant 2^{n+1}-1$, let $\ell_{k}=\frac{\kappa}{2^{n(\gamma-1)}(n+1)^{2}\left(k+1-2^{n}\right)}$, where $\kappa$ is such that $\sum_{n \geqslant 1} \ell_{n}=1$.

For every $n \geqslant 0$ and every $(x, k) \in X$, with $2^{n} \leqslant k \leqslant 2^{n+1}-1$, set $f(x)=D\left(k+1-2^{n}\right)^{\gamma-1}$.

Let $0<\varepsilon \leqslant 1$. We have

$$
\int_{X} f\left(\log ^{+}(f)\right)^{1-\varepsilon} d \mathbb{P} \leqslant C D \sum_{n \geqslant 0} 2^{n(1-\gamma)}(n+1)^{-1-\varepsilon} \sum_{k=2^{n}}^{2^{n+1}-1}\left(k+1-2^{n}\right)^{\gamma-2} \leqslant \tilde{C} D \sum_{n \geqslant 0}(n+1)^{-1-\varepsilon}<\infty .
$$

Taking $D$, large enough, we see that for every $n \geqslant 2, \sum_{k=2^{n-2}}^{2^{n-1}-1} D\left(k+1-2^{n-2}\right)^{\gamma-1}>2^{n \gamma}$. Hence, for that choice of $D$, we infer that $f+\ldots+f \circ \theta^{2^{n}-1}>2^{n \gamma}$ on the set $\cup_{k=1}^{2^{n-1}}\left[0, \ell_{k+2^{n-1}-1}\right] \times\{k\}$. Hence,

$$
\sum_{n \geqslant 0} 2^{n(\gamma-1)} \mathbb{P}\left\{f+\ldots+f \circ \theta^{2^{n}-1}>2^{n \gamma}\right\} \geqslant \sum_{n \geqslant 0} 2^{n(\gamma-1)} \sum_{k=1}^{2^{n-1}} \ell_{k+2^{n-1}-1} \geqslant c \sum_{n \geqslant 0} \frac{1}{n+1}=+\infty
$$

which finishes the proof. 
Proof of Proposition 2.6. Let $\gamma=2 \alpha$. Let $X$ be the probability space constructed in the proof of Lemma 2.5. Let $\Omega_{1}$ be probability space rich enough to support a sequence $\left(\varepsilon_{n}\right)_{n \geqslant 1}$ of i.i.d. $\mathcal{N}(0,1)$ random variables. Let $\Omega:=X \times \Omega_{1}$ with the product measure. Let $f$ be the function satisfying the conclusion of Lemma 2.5. For every $n \geqslant 1$, set $X_{n}:=\varepsilon_{n} f^{1 / 2} \circ \theta^{n}$. Notice that $\left(\varepsilon_{n}\right)_{n \geqslant 1}$ is independent from $\left(f \circ \theta^{n}\right)_{n \geqslant 1}$ so that $\left(X_{n}\right)_{n \geqslant 1}$ is a stationary sequence of martingale differences (and ergodic). Set for every $n \geqslant 1, s_{n}:=\left(\sum_{i=1}^{n} f \circ \theta^{i}\right)^{1 / 2}$. We have, using independence,

$$
\begin{aligned}
\sum_{n \geqslant 0} 2^{n(\alpha-1)} \mathbb{P}\left\{\left|\sum_{i=1}^{2^{n}} X_{i}\right|>2^{n \alpha}\right\} & =\frac{2}{\sqrt{2 \pi}} \sum_{n \geqslant 0} 2^{n(2 \alpha-1)} \mathbb{E}\left[\int_{2^{n \alpha} / s_{2} n}^{+\infty} \mathrm{e}^{-x^{2} / 2} d x\right] \\
& =\frac{2}{\sqrt{2 \pi}} \int_{0}^{+\infty}\left(\sum_{n \geqslant 0} 2^{n(2 \alpha-1)} \mathbb{P}\left\{s_{2^{n}}^{2}>2^{2 n \alpha} / x^{2}\right\}\right) \mathrm{e}^{-x^{2} / 2} d x \\
& \geqslant \frac{2}{\sqrt{2 \pi}}\left(\int_{0}^{1} \mathrm{e}^{-x^{2} / 2} d x\right) \sum_{n \geqslant 0} 2^{n(2 \alpha-1)} \mathbb{P}\left\{s_{2^{n}}^{2}>2^{2 n \alpha}\right\}=+\infty
\end{aligned}
$$

Proof of Theorem 2.8. We apply Theorem1.13 with $r^{\prime}=r, q=2 r$ and $x:=\varepsilon|\mathbf{n}|^{\alpha-1 / r}$ in order to get

$$
\begin{aligned}
& \mathbb{P}\left\{\max _{1 \preccurlyeq \mathbf{i} \preccurlyeq \mathbf{n}}\left\|S_{\mathbf{i}}\right\|>\varepsilon|\mathbf{n}|^{\alpha}\right\} \\
& \qquad C \int_{0}^{+\infty} \mathbb{P}\left\{\left\|X_{\mathbf{1}}\right\|>\varepsilon|\mathbf{n}|^{\alpha-1 / r} u\right\} \min \left\{u^{2 r-1}, u^{r-1}\right\}(1+|\log u|)^{d-1} \mathrm{~d} u .
\end{aligned}
$$

Multiplying by $|\mathbf{n}|^{r \alpha-2}$, summing over $\mathbf{n} \in \mathbb{N}^{d}$ and noticing that for any fixed $N$, the number of elements $\mathbf{k} \in \mathbb{N}^{d}$ such that $\sum_{i=1}^{d} k_{i}=N$ is $c_{d}\left(N^{d-1}+1\right)$ for some constant $c_{d}$ depending only on $d$, we get

$$
\begin{aligned}
& \sum_{\mathbf{n} \in \mathbb{N}^{d}}|\mathbf{n}|^{r \alpha-2} \mathbb{P}\left\{\max _{\mathbf{1} \preccurlyeq \mathbf{i} \preccurlyeq \mathbf{n}}\left\|S_{\mathbf{i}}\right\|>\varepsilon|\mathbf{n}|^{\alpha}\right\} \\
& \leqslant C^{\prime} \int_{0}^{+\infty} \sum_{\mathbf{k} \in \mathbb{N}^{d}} 2^{(r \alpha-1)} \sum_{i=1}^{d} k_{i} \mathbb{P}\left\{\left\|X_{\mathbf{1}}\right\|>\varepsilon 2^{(\alpha-1 / r) \sum_{i=1}^{d} k_{i}} u\right\} \min \left\{u^{2 r-1}, u^{r-1}\right\}(1+|\log u|)^{d-1} \mathrm{~d} u \\
& \leqslant C^{\prime \prime} \int_{0}^{+\infty} \sum_{N=1}^{+\infty} 2^{N(r \alpha-1)} N^{d-1} \mathbb{P}\left\{\left\|X_{1}\right\|>\varepsilon 2^{(\alpha-1 / r) N} u\right\} \min \left\{u^{2 r-1}, u^{r-1}\right\}(1+|\log u|)^{d-1} \mathrm{~d} u
\end{aligned}
$$

and using the fact that for any real valued random variable $Y$,

$$
\sum_{N=1}^{+\infty} 2^{N(r \alpha-1)} N^{d-1} \mathbb{P}\left\{Y>2^{(\alpha-1 / r) N}\right\} \leqslant K_{p, \alpha, r, d} \mathbb{E}\left[Y^{r}(\log (Y))^{d-1} \mathbf{1}\{Y \geqslant 1\}\right],
$$

we are reduced to prove finiteness of

$$
\mathbb{E}\left[\int_{0}^{\frac{\left\|X_{1}\right\|}{\varepsilon}}\left(\frac{\left\|X_{1}\right\|}{u \varepsilon}\right)^{r}\left(\log \left(\frac{\left\|X_{1}\right\|}{u \varepsilon}\right)\right)^{d-1} \min \left\{u^{2 r-1}, u^{r-1}\right\}(1+|\log u|)^{d-1} \mathrm{~d} u\right] .
$$

Let

$$
Y:=\int_{0}^{\frac{\left\|X_{1}\right\|}{\varepsilon}}\left(\frac{\left\|X_{1}\right\|}{u \varepsilon}\right)^{r}\left(\log \left(\frac{\left\|X_{1}\right\|}{u \varepsilon}\right)\right)^{d-1} \min \left\{u^{2 r-1}, u^{r-1}\right\}(1+|\log u|)^{d-1} \mathrm{~d} u .
$$


Assume that $\left\|X_{1}\right\| \leqslant \varepsilon$. Then

$$
Y=\int_{0}^{\frac{\left\|X_{1}\right\|}{\varepsilon}}\left(\frac{\left\|X_{1}\right\|}{u \varepsilon}\right)^{r}\left(\log \left(\frac{\left\|X_{1}\right\|}{u \varepsilon}\right)\right)^{d-1} u^{2 r-1}(1+|\log u|)^{d-1} \mathrm{~d} u
$$

and the substitution $v=u /\left\|X_{1}\right\|$ shows that

$$
Y \leqslant C\left\|X_{1}\right\|^{2 r}\left(1-\log \left(\left\|X_{1}\right\|\right)\right)^{d-1} .
$$

Now if we assume that $\left\|X_{1}\right\|>\varepsilon$, then

$$
\begin{aligned}
Y=\int_{0}^{1}\left(\frac{\left\|X_{\mathbf{1}}\right\|}{u \varepsilon}\right)^{r}(\log ( & \left.\left.\frac{\left\|X_{\mathbf{1}}\right\|}{u \varepsilon}\right)\right)^{d-1} u^{2 r-1}(1+|\log u|)^{d-1} \mathrm{~d} u \\
& +\int_{1}^{\frac{\left\|X_{\mathbf{1}}\right\|}{\varepsilon}}\left(\frac{\left\|X_{\mathbf{1}}\right\|}{u \varepsilon}\right)^{r}\left(\log \left(\frac{\left\|X_{\mathbf{1}}\right\|}{u \varepsilon}\right)\right)^{d-1} u^{r-1}(1+|\log u|)^{d-1} \mathrm{~d} u
\end{aligned}
$$

and the first term of the right-hand-side can be controlled by $C\left\|X_{1}\right\|^{r}\left(1+\left|\log \left\|X_{1}\right\|\right|\right)^{d-1}$, while for the second, the substitution $t:=\log u$ and an integration by parts yield $Y \leqslant C\left\|X_{1}\right\|^{r}\left(1+\left|\log \left\|X_{1}\right\|\right|\right)^{d}$. We thus got the estimate

$$
Y \leqslant C\left\|X_{1}\right\|^{r}\left(1+\left|\log \left\|X_{1}\right\|\right|\right)^{d}
$$

where $C$ depends only on $\varepsilon, d$ and $r$. Since $X_{1}$ belongs to $\mathbb{L}^{r} \log ^{d} \mathbb{L}$, we proved (2.13) and the proof of Theorem 2.10 is finished.

Proof of Theorem 2.10. We apply Theorem1.13 with $r^{\prime}=2, q=2 p$ and $x:=\varepsilon|\mathbf{n}|^{\alpha-1 / 2}$ in order to get

$$
\begin{aligned}
\mathbb{P}\left\{\max _{1 \preccurlyeq \mathbf{i} \preccurlyeq \mathbf{n}}\left\|S_{\mathbf{i}}\right\|>\varepsilon|\mathbf{n}|^{\alpha}\right\} & \\
& \leqslant C \int_{0}^{+\infty} \mathbb{P}\left\{\left\|X_{\mathbf{1}}\right\|>\varepsilon|\mathbf{n}|^{\alpha-1 / 2} u\right\} \min \left\{u^{2 p-1}, u\right\}(1+|\log u|)^{d-1} \mathrm{~d} u .
\end{aligned}
$$

Multiplying by $|\mathbf{n}|^{p(\alpha-1 / 2)-1}$, summing over $\mathbf{n} \in \mathbb{N}^{d}$ and noticing that for any fixed $N$, the number of elements $\mathbf{k} \in \mathbb{N}^{d}$ such that $\sum_{i=1}^{d} k_{i}=N$ is $c_{d}\left(N^{d-1}+1\right)$ for some constant $c_{d}$ depending only on $d$, we get

$$
\begin{aligned}
& \sum_{\mathbf{n} \in \mathbb{N}^{d}}|\mathbf{n}|^{p(\alpha-1 / 2)-1} \mathbb{P}\left\{\max _{\mathbf{1} \preccurlyeq \mathbf{i} \preccurlyeq \mathbf{n}}\left\|S_{\mathbf{i}}\right\|>\varepsilon|\mathbf{n}|^{\alpha}\right\} \\
& \leqslant C^{\prime} \int_{0}^{+\infty} \sum_{\mathbf{k} \in \mathbb{N}^{d}} 2^{(p(\alpha-1 / 2)-1) \sum_{i=1}^{d} k_{i}} \mathbb{P}\left\{\left\|X_{\mathbf{1}}\right\|>\varepsilon 2^{(\alpha-1 / 2) \sum_{i=1}^{d} k_{i}} u\right\} \min \left\{u^{2 p-1}, u\right\}(1+|\log u|)^{d-1} \mathrm{~d} u \\
& \leqslant C^{\prime \prime} \int_{0}^{+\infty} \sum_{N=1}^{+\infty} 2^{N p(\alpha-1 / 2)} N^{d-1} \mathbb{P}\left\{\left\|X_{\mathbf{1}}\right\|>\varepsilon 2^{(\alpha-1 / p) N} u\right\} \min \left\{u^{2 p-1}, u\right\}(1+|\log u|)^{d-1} \mathrm{~d} u
\end{aligned}
$$

and using the fact that for any real valued random variable $Y$,

$$
\sum_{N=1}^{+\infty} 2^{N p(\alpha-1 / 2)} N^{d-1} \mathbb{P}\left\{Y>2^{(\alpha-1 / 2) N}\right\} \leqslant K_{p, \alpha, r, d} \mathbb{E}\left[Y^{p}(\log (Y))^{d-1} \mathbf{1}\{Y \geqslant 1\}\right],
$$

we are reduced to prove finiteness of

$$
\mathbb{E}\left[\int_{0}^{\frac{\left\|X_{\mathbf{1}}\right\|}{\varepsilon}}\left(\frac{\left\|X_{\mathbf{1}}\right\|}{u \varepsilon}\right)^{p}\left(\log \left(\frac{\left\|X_{\mathbf{1}}\right\|}{u \varepsilon}\right)\right)^{d-1} \min \left\{u^{2 p-1}, u\right\}(1+|\log u|)^{d-1} \mathrm{~d} u\right] .
$$


Let

$$
Y:=\int_{0}^{\frac{\left\|X_{1}\right\|}{\varepsilon}}\left(\frac{\left\|X_{\mathbf{1}}\right\|}{u \varepsilon}\right)^{p}\left(\log \left(\frac{\left\|X_{\mathbf{1}}\right\|}{u \varepsilon}\right)\right)^{d-1} \min \left\{u^{2 p-1}, u\right\}(1+|\log u|)^{d-1} \mathrm{~d} u .
$$

If $\left\|X_{1}\right\| / \varepsilon \leqslant 1$, then

$$
\begin{aligned}
& Y=\int_{0}^{\frac{\left\|X_{\mathbf{1}}\right\|}{\varepsilon}}\left(\frac{\left\|X_{\mathbf{1}}\right\|}{u \varepsilon}\right)^{p}\left(\log \left(\frac{\left\|X_{\mathbf{1}}\right\|}{u \varepsilon}\right)\right)^{d-1} u^{2 p-1}(1+|\log u|)^{d-1} \mathrm{~d} u \\
& \leqslant C\left\|X_{\mathbf{1}}\right\|^{2 p}\left(1+\left|\log \left\|X_{\mathbf{1}}\right\|\right|\right)^{d-1} .
\end{aligned}
$$

If $\left\|X_{1}\right\| / \varepsilon>1$, then

$$
\begin{gathered}
Y=\int_{0}^{1}\left(\frac{\left\|X_{\mathbf{1}}\right\|}{u \varepsilon}\right)^{p}\left(\log \left(\frac{\left\|X_{\mathbf{1}}\right\|}{u \varepsilon}\right)\right)^{d-1} u^{2 p-1}(1+|\log u|)^{d-1} \mathrm{~d} u \\
\quad+\int_{1}^{\left\|X_{\mathbf{1}}\right\|}\left(\frac{\left\|X_{\mathbf{1}}\right\|}{u \varepsilon}\right)^{p}\left(\log \left(\frac{\left\|X_{\mathbf{1}}\right\|}{u \varepsilon}\right)\right)^{d-1} u(1+|\log u|)^{d-1} \mathrm{~d} u .
\end{gathered}
$$

The first term can be bounded by $C\left\|X_{1}\right\|^{p}\left(1+\left|\log \left\|X_{1}\right\|\right|\right)^{d-1}$ and for the second one, the substitution $t:=\log u$ shows that a similar upper bound can be given. This ends the proof of Theorem 2.10.

Acknowledgment The author would like to thank Christophe Cuny for giving the statement and proof of Lemma 2.5 and Proposition 2.6.

\section{REFERENCES}

[Als90] G. Alsmeyer, Convergence rates in the law of large numbers for martingales, Stochastic Process. Appl. 36 (1990), no. 2, 181-194. MR 10849747

[Ass75] P. Assouad, Espaces p-lisses et q-convexes, inégalités de Burkholder, 8. MR 04079632

[Azu67] K. Azuma, Weighted sums of certain dependent random variables, Tôhoku Math. J. (2) 19 (1967), 357-367. MR 02215711

[BK65] L. E. Baum and M. Katz, Convergence rates in the law of large numbers, Trans. Amer. Math. Soc. 120 (1965), 108-123. MR 019852418

[Bur73] D. L. Burkholder, Distribution function inequalities for martingales, Ann. Probability 1 (1973), $19-42$. MR 03656921,8

[dlPn99] V. H. de la Peña, A general class of exponential inequalities for martingales and ratios, Ann. Probab. 27 (1999), no. 1, 537-564. MR 16811531

[DM07] J. Dedecker and F. Merlevède, Convergence rates in the law of large numbers for Banach-valued dependent variables, Teor. Veroyatn. Primen. 52 (2007), no. 3, 562-587. MR 2743029 6, 8

[Faz05] I. Fazekas, Burkholder's inequality for multiindex martingales, Ann. Math. Inform. 32 (2005), 45-51. MR 22648665

[FGL12] X. Fan, I. Grama, and Q. Liu, Large deviation exponential inequalities for supermartingales, Electron. Commun. Probab. 17 (2012), no. 59, 8. MR 30057321

[FGL15] _ Exponential inequalities for martingales with applications, Electron. J. Probab. 20 (2015), no. 1, 22. MR 33112141

[FGL17] _ Deviation inequalities for martingales with applications, J. Math. Anal. Appl. 448 (2017), no. 1, 538-566. MR 35798986

[Gut78] A. Gut, Marcinkiewicz laws and convergence rates in the law of large numbers for random variables with multidimensional indices, Ann. Probability 6 (1978), no. 3, 469-482. MR 04944318

[Hae84] E. Haeusler, An exact rate of convergence in the functional central limit theorem for special martingale difference arrays, Z. Wahrsch. Verw. Gebiete 65 (1984), no. 4, 523-534. MR 7361441

[Hao13] S. Hao, Convergence rates in the law of large numbers for arrays of Banach valued martingale differences, Abstr. Appl. Anal. (2013), Art. ID 715054, 26. MR 31325447

[HL14] Shunli Hao and Quansheng Liu, Convergence rates in the law of large numbers for arrays of martingale differences, J. Math. Anal. Appl. 417 (2014), no. 2, 733-773. MR 31945127

[Hoe63] W. Hoeffding, Probability inequalities for sums of bounded random variables, J. Amer. Statist. Assoc. 58 (1963), 13-30. MR 01443631

[JS88] W. B. Johnson and G. Schechtman, Martingale inequalities in rearrangement invariant function spaces, Israel J. Math. 64 (1988), no. 3, 267-275 (1989). MR 9955725

[Kho02] D. Khoshnevisan, Multiparameter processes, Springer Monographs in Mathematics, Springer-Verlag, New York, 2002, An introduction to random fields. MR 19147484 
[KL11] A. Kuczmaszewska and Z. A. Lagodowski, Convergence rates in the SLLN for some classes of dependent random fields, J. Math. Anal. Appl. 380 (2011), no. 2, 571-584. MR 27944158

[Lag16] Z. A. Lagodowski, An approach to complete convergence theorems for dependent random fields via application of Fuk-Nagaev inequality, J. Math. Anal. Appl. 437 (2016), no. 1, 380-395. MR 34519718

[LV01] E. Lesigne and D. Volný, Large deviations for martingales, Stochastic Process. Appl. 96 (2001), no. 1, 143-159. MR 18566848

[Nag03] S. V. Nagaev, On probability and moment inequalities for supermartingales and martingales, Proceedings of the Eighth Vilnius Conference on Probability Theory and Mathematical Statistics, Part II (2002), vol. 79, 2003, pp. 35-46. MR 2021875 1, 2, 3

[Pis75] G. Pisier, Martingales with values in uniformly convex spaces, Israel J. Math. 20 (1975), no. 3-4, 326-350. MR 0394135 2, 8

[Rue81] L. Rüschendorf, Ordering of distributions and rearrangement of functions, Ann. Probab. 9 (1981), no. 2, 276-283. MR 606989 12, 13

Ruhr-Universität Bochum Fakultät FÜr Mathematik NA 3/32 Universitätsstrasse 15044780 Bochum,.

E-mail address: Davide.Giraudo@ruhr-uni-bochum.de 